IZA DP No. 5027

Optimal Incentive Contracts under Moral Hazard When the Agent Is Free to Leave

Florian Englmaier

Gerd Muehlheusser

Andreas Roider

June 2010 


\title{
Optimal Incentive Contracts under Moral Hazard When the Agent Is Free to Leave
}

\author{
Florian Englmaier
}

University of Munich

\author{
Gerd Muehlheusser \\ University of Bielefeld \\ and IZA
}

\author{
Andreas Roider \\ University of Heidelberg \\ and IZA
}

\section{June 2010 \\ IZA \\ P.O. Box 7240 \\ 53072 Bonn \\ Germany}

Discussion Paper No. 5027

Phone: +49-228-3894-0

Fax: +49-228-3894-180

E-mail: iza@iza.org

\begin{abstract}
Any opinions expressed here are those of the author(s) and not those of IZA. Research published in this series may include views on policy, but the institute itself takes no institutional policy positions.

The Institute for the Study of Labor (IZA) in Bonn is a local and virtual international research center and a place of communication between science, politics and business. IZA is an independent nonprofit organization supported by Deutsche Post Foundation. The center is associated with the University of Bonn and offers a stimulating research environment through its international network, workshops and conferences, data service, project support, research visits and doctoral program. IZA engages in (i) original and internationally competitive research in all fields of labor economics, (ii) development of policy concepts, and (iii) dissemination of research results and concepts to the interested public.
\end{abstract}

IZA Discussion Papers often represent preliminary work and are circulated to encourage discussion. Citation of such a paper should account for its provisional character. A revised version may be available directly from the author. 
IZA Discussion Paper No. 5027

June 2010

\section{ABSTRACT \\ Optimal Incentive Contracts under Moral Hazard When the Agent Is Free to Leave ${ }^{*}$}

We characterize optimal incentive contracts in a moral hazard framework extended in two directions. First, after effort provision, the agent is free to leave and pursue some ex-post outside option. Second, the value of this outside option is increasing in effort, and hence endogenous. Optimal contracts may entail properties such as inducing first-best effort and surplus, or non-responsiveness with respect to changes in verifiable parameters. Moreover, while always socially inefficient, separation might occur in equilibrium. Except for the latter, these findings are robust to renegotiation. When the outside option is exogenous instead, the standard results obtain.

JEL Classification: D86, D82, K31, M52

Keywords: moral hazard, limited commitment, ex-post outside option, limited liability

Corresponding author:

Andreas Roider

Department of Economics

University of Heidelberg

Bergheimer Str. 58

69115 Heidelberg

Germany

E-mail: roider@uni-hd.de

\footnotetext{
*We would like to thank Mathias Dewatripont, Isadora Dorn, Oliver Hart, Matthias Kräkel, Patrick Schmitz, and seminar participants at Bonn, Cologne, Dortmund, Heidelberg, Mannheim, the 2010 CEPR conference on "Science, Innovation, Firms, and Markets in a Globalized World" in Budapest, the 2009 conference on "Contracts, Firms, and Corporate Finance" in Paris, and the 2009 SFB/TR15conference in Tutzing for helpful comments and discussions. Part of this research was conducted at Harvard Business School and CES Munich, whose hospitality the first author, respectively the second and third authors gratefully acknowledge. We would also like to thank the German Science Foundation for financial support through SFB/TR 15 "Governance and the Efficiency of Economic Systems".
} 


\section{Introduction}

Motivation In the standard moral hazard model, participation of the agent throughout the relationship with the principal is taken for granted. That is, after a contract is signed, the agent chooses his effort, subsequently some output is realized, and finally the agent receives payments as stipulated by the contract. However, the principal-agent literature has identified a number of contexts, employment relationships in particular, where despite of a formal contract with the principal, agents are basically free to leave at any time (see e.g., Harris and Holmstrom, 1982; Holmstrom, 1983; Meyer, 1987; Phelan, 1995; Krueger and Uhlig, 2006). Potential factors contributing to this phenomenon include the employment-at-will doctrine in U.S. employment law or difficulties in the course of trying to enforce participation on an unwilling employee (see e.g., Malcomson, 1999, p. 2304f.).

For the principal, the agent's freedom to leave might be a concern when both the agent's effort and his subsequent presence are essential for project success. At the same time, in many settings, more effort within the relationship with the principal will improve the agent's outside opportunities. For example, in the course of working on the project, the agent might accumulate crucial human capital that he may also profitably use outside his relationship with the principal (e.g., by starting a new business). If this is the case, the principal will have to take into account that providing high effort incentives might make it more attractive for the agent to leave in the middle of the project; thereby endangering the project return. As discussed below, such issues are particularly relevant for "knowledge workers" (Drucker, 1959) whose human capital is crucial for the principal (see e.g., Pakes and Nitzan, 1983; Anton and Yao, 1995; Lerner and Malmendier, 2010).

In such a context, the following questions arise naturally: How should optimal incentive contracts be structured in settings where contracts must be sufficiently attractive to retain the agent? Might it be optimal for the principal to induce separation even if this is socially inefficient? Does the additional source of frictions imposed by the agent's freedom to leave necessarily have a negative welfare effect? 
Framework To address these questions, we employ a workhorse moral hazard model (riskneutrality, limited liability, and binary output space) and modify it in the following two ways. ${ }^{1}$ First, while the principal can commit to the contract terms (i.e., output-dependent payments), the agent is free to leave. ${ }^{2}$ In particular, after effort provision the agent learns the realized state of the world and may opt to separate from the principal in order to pursue some ex-post outside option. Second, we focus on the case where this ex-post outside option is increasing in the agent's chosen effort, and hence endogenous. ${ }^{3}$

Compared to the standard model, these additional features have a non-trivial impact on the incentive structure. In particular, the agent's ex-post participation decision will not only depend on the terms of the initial contract (which determines his payoff when staying with the principal), but may also depend on his chosen effort level (which, via the value of his ex-post outside option, determines his payoff when leaving). As a result, the agent's optimal effort and ex-post participation decisions are intertwined, which, compared to the standard model, makes the principal's contract design problem substantially more intricate. Consequently, the main contribution of this paper is the characterization of optimal incentive contracts in this setting.

Results We show that the contract optimally offered by the principal is either one of two types: under a no-separation contract all specified payments are sufficiently large such that the agent is induced to always stay, which is also the ex-post efficient decision. Alternatively, under a partial-separation contract, the agent is induced to leave in the low state of the world.

The exact properties of optimal no-separation contracts depend on the size of the first-best effort level and thus on how strongly effort affects expected surplus. In particular, there are three relevant cases, each of which may emerge in equilibrium. First, as long as the first-best effort level is not too large, it is optimally induced by the principal, and the first-best surplus is realized. In this case, from a welfare perspective, the additional friction imposed by the agent's freedom to leave in fact neutralizes the frictions imposed by unobservable effort and limited

\footnotetext{
${ }^{1}$ See e.g., Laffont and Martimort (2002, pp. 194ff.) for a textbook treatment and Tirole (1999) for an application in the context of corporate governance.

${ }^{2}$ This is in line with the literature on one-sided commitment discussed above, which argues that in many settings (such as employment, financing, or insurance), principals will find it easier to commit to initial contract terms; legally or by other means such as reputation (see e.g., Krueger and Uhlig, 2006).

${ }^{3}$ In the following, we will frequently omit the term "ex-post" and simply refer to the agent's "outside option". As will become clear, participation ex-ante is always ensured, and hence not an issue.
} 
liability alone. ${ }^{4}$ Second, for intermediate levels of first-best effort, the principal optimally offers exactly the same contract (inducing also the same effort level) in this entire range of parameters. Hence, while we are in a complete-contracting framework, an outside observer may perceive the optimal contract as incomplete in the sense of being non-responsive to changes in the verifiable output (for a detailed discussion of incomplete contracts and their foundation, see e.g., Tirole, 1999). Third, for sufficiently large first-best effort levels, the implemented effort is again increasing in first-best effort, but it is inefficiently low and, compared to the standard model with full commitment, the downward distortion is even larger.

Clearly, the principal can always ensure no-separation by stipulating sufficiently attractive payments. However, we show that for large levels of first-best effort, the principal may strictly prefer to offer a partial-separation contract, which induces the agent to leave when the low state of the world is realized. This result holds even though we assume that in case of separation, the principal does not benefit at all from the agent's effort. Moreover, separation is also socially inefficient. ${ }^{5}$ Our partial-separation result is reminiscent of up-or-out policies, as frequently used in human-capital intensive industries, such as law or consulting firms (see e.g., Gibbons and Waldmann, 1999; O'Flaherty and Siow, 1995). Such policies are often enforced informally, for example by offering an employee a very low bonus or by moving him to a less intriguing job, in order to induce him to leave "voluntarily". As a further interesting feature of partial-separation contracts, the agent may in fact be harmed if his ex-post outside option becomes ceteris paribus more attractive.

Finally, we show that our results are driven by the combination of the agent's freedom to leave and the endogeneity of his outside option. To this end, we consider a model variant where the agent is free to leave, but where his outside option is exogenous, i.e., independent of the chosen effort (such that his subsequent participation decision will not be affected by his effort choice). Compared to the standard model with full-commitment, the payments under the optimal contract are then simply scaled up by the value of the outside option, but otherwise the same effort and participation decisions obtain.

\footnotetext{
${ }^{4}$ In some cases, (employment) contracts stipulate non-compete clauses with the purpose of preventing agents from using their acquired skills outside the relationship. However, as discussed in section 5, courts are often reluctant to enforce such clauses, which, from a welfare perspective, our result seems to corroborate.

${ }^{5}$ However, as discussed in section 5 , if renegotiation cannot be ruled out, without loss of generality, one can restrict attention to no-separation contracts, and all of our results with respect to this type of contract remain intact.
} 
Intuition One crucial difference between the exogenous and endogenous outside option cases is that in the former, the principal can avoid leaving a rent to the agent at least in some states of the world (i.e., the agent is then indifferent between staying and leaving in those states). In the latter case of an endogenous outside option, however, the agent will in general respond to any given contract by choosing an effort level such that ex-post, he either strictly prefers to stay or strictly prefers to leave, which implies that he will earn a rent in each state in which he stays with the principal. Consequently, inducing no-separation is more costly for the principal, and the agent's rent has properties qualitatively different from both the standard model and the exogenous outside option case.

The optimal no-separation contract makes the agent indifferent between choosing an effort level that subsequently induces him to stay in all states and choosing a different effort level that (depending on parameters) subsequently induces him to either (i) leave in all states or (ii) leave in the low state only. In the former case, the agent's payoff (and hence, his rent) is invariant in the terms of the contract, which makes the principal the residual claimant, who then finds it optimal to provide first-best incentives. In the latter case, the agent's rent is increasing in effort, which leads the principal to optimally distort the induced effort below first-best, and potentially even below the effort level that would obtain in the standard model; the reason being that the agent's marginal rent has an additional term to ensure that separation does not occur. Moreover, at the boundary between these two cases the agent's marginal rent makes a discrete jump from zero to strictly positive, and so does the principal's marginal virtual cost of inducing a given effort level. Consequently, even as the marginal benefit of more effort increases, there exists a parameter range where the principal keeps the implemented effort level constant, resulting in non-responsiveness of the optimal contract in that range.

When the effort level that the principal intends to implement is large, inducing no-separation is very costly for the principal because, in this case, the agent's rent is increasing in effort. Hence, as it allows to reduce the agent's rent, the principal might prefer to induce partialseparation; thereby foregoing the output in the low state. In this case, the principal free-rides on the (external) effort incentives generated by the agent's endogenous outside option. Moreover, the effort incentives generated through the outside option respectively the contract may be substitutes, and hence a more attractive outside option may lead the principal to reduce the contractual payments, which, in total, might even reduce the agent's expected payoff. 
Related literature To the best of our knowledge, this is the first paper to consider optimal incentive contracts in a moral hazard framework where the agent is free to exercise some (ex-post) outside option that is endogenous (i.e., determined by the effort chosen inside the contractual relationship with the principal). Of course, these important features have been addressed previously, but in contexts differing from ours.

There is an early strand of research on labor markets with one-sided commitment where the agent is free to leave but the principal can commit to the contract terms (for an overview, see e.g., Meyer, 1987). For example, in order to analyze career wage profiles, Harris and Holmstrom (1982) and Holmstrom (1983) study optimal insurance problems in the context of dynamic principal-agent setups where ex-ante effort provision plays, however, no role. ${ }^{6}$ More closely related, in one of the first papers to discuss limited liability in a (static) moral hazard framework, Sappington (1983) considers a setting with hidden information: after having entered the relationship with the principal, the agent receives private information on a productivity shock and may decide to leave the principal before providing effort.

Furthermore, a prominent strand in the one-sided commitment literature deals with labor mobility; studying situations where "knowledge workers" (such as scientists or engineers) may walk away in the course of a project (e.g., in order to start their own business), which might endanger the principal's project return. For example, in a seminal paper, Pakes and Nitzan (1983) investigate optimal separation decisions of firms and workers in a complete information framework where effort is fixed; resulting in efficient retention decisions in all possible states of the world. Building on Pakes and Nitzan (1983), Anton and Yao (1995) also abstract from effort provision, but analyze a situation where the agent has private information on the value of an innovation (which, when leaving the principal, he may exploit to form a start-up business). They show that there might be inefficient separation ex-post, the extent of which depends on the degree of asset complementarity and the nature of competition. In a recent paper, Lerner and Malmendier (2010) study a phenomenon that is related to issues of labor mobility but does not necessarily imply that the agent (completely) leaves the principal. For the biotechnology industry, Lerner and Malmendier (2010) document that "project substitution" seems to be a major concern of financing firms, who worry that (instead of solely focussing on completing the

\footnotetext{
${ }^{6}$ Phelan (1995) and Krueger and Uhlig (2006) consider related setups to study optimal dynamic consumption profiles. Note that in Krueger and Uhlig (2006) the term "endogenous outside option" is used to describe an outside option whose value is determined by competition among principals.
} 
project) start-up entrepreneurs may spend time and resources on work for alternative alliances, own products, or even academic papers (leading to a dissipation of the project returns). Thus, project substitution may be seen as a milder version of our setting where, upon the agent's departure, the project return is lost completely.

With respect to endogenous outside options, potential effects of effort on an agent's outside opportunities have not received much attention in the moral hazard literature (for a recent exception, see e.g., Netzer and Scheuer, 2008). However, such potential outside effects are a central feature in the literature on career concerns (see e.g., Holmstrom, 1999; Dewatripont, Jewitt, and Tirole, 1999). There, over time, the principal (and potential alternative employers) receive information about the agent's unobservable productivity type by observing output (which depends on both the agent's type and his unobservable effort). In the simplest version, there are no output-contingent contracts, and in each period the agent's outside option is based on his assessed ability. ${ }^{7}$ Consequently, through his current effort choice, the agent aims to influence the principal's (and the outside market's) learning process. More closely related, Gibbons and Murphy (1992) consider the interaction of internal incentives (through explicit incentive contracts) and market-based incentives (through career concerns) to study their relative importance over the career horizon.

The remainder of the paper is structured as follows. Section 2 introduces the model and the first-best benchmark. Section 3 derives optimal agent behavior for a given contract. Section 4 contains our main results: we characterize optimal no-separation contracts and optimal partialseparation contracts, as well as the principal's ultimate choice between the two. Section 5 in turn discusses non-compete clauses, the exogenous outside option case as well as two robustness checks: we show that the set of contracts considered is without loss of generality, and we discuss the issue of renegotiation. Section 6 concludes. All proofs are relegated to the Appendix.

\section{Model}

Basic framework We consider an extension of a standard moral-hazard model with a principal (she) and an agent (he), who are both risk-neutral and where the agent faces a limited-

\footnotetext{
${ }^{7}$ Note that, similar to the present paper, in career concern models the agent is in principle free to leave, but the focus is on potential separation after output has been realized.
} 


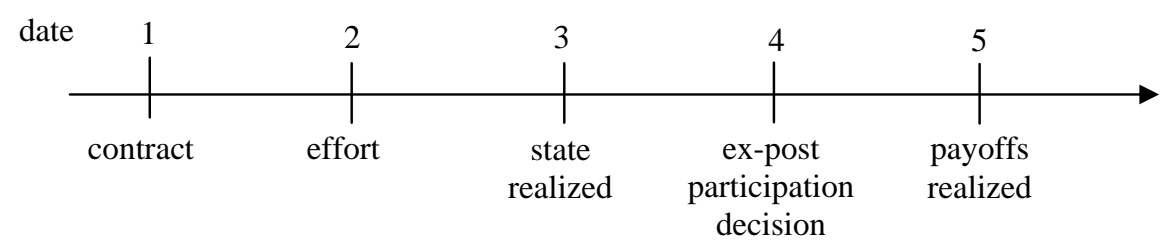

Figure 1: Sequence of events

liability constraint. ${ }^{8}$ The sequence of events is as follows (see Figure 1): The principal needs the agent to conduct some project and proposes (take-it-or-leave-it) a contract to the agent at date 1 (feasible contracts will be discussed below). When deciding whether or not to accept the offer, we follow the standard model in that the agent's (ex-ante) reservation utility is a constant, which we set to zero.

At date 2 , the agent exerts unobservable effort $e \in[0,1]$, which stochastically influences which state of the world $s \in\{\underline{s}, \bar{s}\}$ is realized at date 3 , where $\bar{s}>\underline{s}>0$, and where $\Delta s \equiv \bar{s}-\underline{s}$. In particular, we assume $\operatorname{Prob}(s=\bar{s})=e$ and $\operatorname{Prob}(s=\underline{s})=1-e$, so that higher effort makes $s=\bar{s}$ more likely. Effort costs are given by $c(e)=\frac{1}{2} e^{2}$ for simplicity. ${ }^{9}$

In addition, the agent's effort choice not only affects the expected surplus of the relationship with the principal, but also the value $v(e)$ of the agent's ex-post outside option that he might pursue at date 4 after having privately learned the state $s$ at date 3 . As discussed above, the agent might, for example, acquire some knowledge in the course of the project that he can also profitably use outside the relationship with the principal, i.e., $v^{\prime}(e)>0$. In what follows, let $v(e)=\lambda e$ so that the parameter $\lambda>0$ measures the intensity of the agent's effort on his ex-post outside option. Moreover, we assume $\lambda<\underline{s}$ such that, independent of $e$, separation is always inefficient ex-post.

We assume that the principal only reaps a profit from the project if the agent decides to stay at date 4, i.e., the presence of the agent is essential for the principal to realize a profit at all. This assumption makes separation very harmful for the principal. Nevertheless, as shown below, the principal might find it optimal to induce separation, which, however, is inefficient. Formally, if the agent stays (i.e., "completes the project"), the principal's verifiable gross profit at date 5 is simply $s$. If the agent leaves, the principal's gross profit is zero.

\footnotetext{
${ }^{8}$ Note that, in the absence of limited liability, the first-best outcome can easily be implemented, for example, by a sell-the-shop contract.

${ }^{9}$ While at some points this assumption simplifies the analysis, it is not crucial for our main results.
} 
Informational assumptions and contract space Since the agent's effort $e$ is unobservable, his ex-post outside option is non-contractible (which is line with the literature on knowledge workers). In contrast, the gross profit realized by the principal at date 5 (which is $\underline{s}, \bar{s}$, or 0 in case the agent leaves) is observable and verifiable. Therefore, the initial contract may specify payments that may depend on the realized gross profit and that, due to the agent's limited liability, have to be non-negative. In principle, the contract may also condition on messages that, after date 3 , the agent sends about his effort level and the realized state. However, we show in section 5 that, akin to the standard moral hazard model, for any incentive-compatible message-dependent contract there exists a contract that does not condition on messages and leads to the same equilibrium outcome (i.e., effort level, ex-post participation decisions, and payoffs). Moreover, as is also shown in section 5, from the principal's perspective, it can neither be optimal to promise a positive payment if the gross profit is zero (i.e., if the agent leaves) nor to specify a payment when $\underline{s}$ is realized that exceeds the payment when $\bar{s}$ is realized. Hence, without loss of generality, in the following we restrict attention to contracts of the form $t \in T^{+} \equiv\{(\underline{t}, \bar{t}) \mid \bar{t} \geq \underline{t} \geq 0\}$, where $\bar{t}$ and $\underline{t}$ denote the transfers to the agent if the realized gross profit is given by $\bar{s}$ and $\underline{s}$, respectively.

As discussed in the introduction, we focus on a situation where there is one-sided commitment only: while the principal can commit to the payments specified in the contract, the agent cannot be forced (or simply promise) to stay. That is, contracts that prohibit the agent to leave at intermediate stages of the project are not feasible. Consequently, if the principal intends to induce the participation of the agent throughout the entire project, she must structure the contractually promised payments in a way that gives the agent an incentive to stay.

Payoffs Given the discussion above, from an ex-ante perspective, the principal's expected payoff is

$$
e \cdot(\bar{s}-\bar{t}) \cdot \bar{I}+(1-e) \cdot(\underline{s}-\underline{t}) \cdot \underline{I},
$$

while for the agent we have

$$
e \cdot[\bar{t} \cdot \bar{I}+v(e) \cdot(1-\bar{I})]+(1-e) \cdot[\underline{t} \cdot \underline{I}+v(e) \cdot(1-\underline{I})]-c(e)
$$

where $\bar{I}$ and $\underline{I}$ are indicator variables that are equal to 1 if, at date 4 , the agent decides to stay with the principal in state $\bar{s}$ and $\underline{s}$, respectively, and that are zero otherwise. Note that 
(1) and (2) immediately imply that ex-ante participation is not an issue. That is, the principal can always secure herself a payoff of at least zero by proposing the contract $\underline{t}=\bar{t}=0$, while the agent can secure himself a payoff of at least zero by choosing $e=0$.

First-best Note that the assumption $\lambda<\underline{s}$ together with $e \in[0,1]$ implies $v(e)<s$ for all $e$ and $s$, and hence, from a first-best perspective, the agent should never leave the principal at stage 4. Consequently, the first-best effort level $e^{F B}$ is given by

$$
e^{F B}(\Delta s) \equiv \underset{e}{\operatorname{argmax}}\left\{e \bar{s}+(1-e) \underline{s}-\frac{1}{2} e^{2}\right\}=\min (\Delta s, 1),
$$

and, as $e=0$ is feasible, the resulting first-best social surplus is strictly positive. Note that the first-best effort level depends on the spread $\Delta s=\bar{s}-\underline{s}$ only.

\section{Optimal agent behavior for a given contract}

In this section, we characterize the optimal agent behavior for a given contract $t$, where we proceed in four steps. In step 1, we derive the agent's optimal ex-post participation decision at date 4 (Lemma 1). This decision will, in general, depend on both the contract and the previously chosen effort level: the latter affects the value of the agent's outside option, which in combination with the contract terms is crucial for his (optimal) decision to stay or leave. It turns out that, when taking into account the agent's optimal ex-post participation decision, the functional form of his payoff $U(e, t)$ varies across the contract space. Consequently, in order to ultimately derive the agent's optimal effort level, in a preliminary step 2, we characterize $U(e, t)$ (Lemma 2). In step 3, we establish that the number of candidate optimal effort level is limited (Lemma 3). Finally, in step 4, we show that the contract space can be partitioned in three subsets of contracts that differ with respect to the agent's ex-post participation decisions that emerge in equilibrium. In combination with our previous results, this will then allow to fully characterize the agent's optimal behavior for any given contract (Proposition 1).

Step 1: Ex-post participation At date 4, the state $s$ is already known to the agent, so that his ex-post participation decision depends on the respective payment $\underline{t}$ or $\bar{t}$ as stipulated in the contract as well as the previously chosen effort level (which leads to an outside option of $\lambda e$ ). Clearly, for $s$ given, the agent will optimally stay as long as the payment from the 
principal (weakly) exceeds the agent's outside option. Hence, in state $\underline{s}(\bar{s})$ the agent optimally stays if $\underline{t} \geq \lambda e(\bar{t} \geq \lambda e)$ holds. ${ }^{10}$ Put differently, in state $\underline{s}(\bar{s})$, the agent optimally stays if and only if the previously chosen effort level is weakly below some threshold value, i.e., if $e \leq \frac{t}{\lambda}$ $\left(e \leq \frac{\bar{t}}{\lambda}\right)$ holds. Note that $\bar{t} \geq \underline{t}$ implies that it can never be optimal for the agent to stay when $s=\underline{s}$, but to leave when $s=\bar{s}$.

Lemma 1 (Optimal ex-post participation at date 4) For a given contract $t \in T^{+}$, the agent's optimal participation decision at date 4 may depend on the previously chosen effort level e. In particular,

(i) for low effort levels $e \in E_{2}(t) \equiv\left\{e \mid e \leq \frac{t}{\lambda}\right\}$, he stays in both states,

(ii) for intermediate effort levels $e \in E_{1}(t) \equiv\left\{e \mid \frac{t}{\bar{\lambda}}<e \leq \frac{\bar{t}}{\lambda}\right\}$, he stays in state $\bar{s}$ only, and

(iii) for high effort levels $e \in E_{0}(t) \equiv\left\{e \mid e>\frac{\bar{t}}{\lambda}\right\}$, he stays in neither state.

Note that, depending on the underlying contract $t$, the sets $E_{1}(t)$ and $E_{0}(t)$ might be empty (see Figure 2). That is, for a given contract $t$, independent of the chosen effort level, the respective ex-post participation decision(s) will not occur in equilibrium. Put differently, when taking into account the agent's optimal ex-post participation decisions, in each of the cases (i), (ii), and (iii) of Figure 2 the functional form of the agent's payoff (see (2))

$$
U(e, t) \equiv e \cdot \max (\bar{t}, \lambda e)+(1-e) \cdot \max (\underline{t}, \lambda e)-c(e)
$$

will be different, and its exact functional form depends on the underlying contract $t$. This issue is crucial for determining the agent's optimal effort choice and will be considered next.

Step 2: Characterizing the agent's payoff function To determine which of the sets $E_{0}(t), E_{1}(t)$, and $E_{2}(t)$ are non-empty, note that (except for knife-edge cases) one of the following three situations may emerge for a given contract (see Figure 2): First, for the type of contract depicted in panel (i) both critical values $\frac{t}{\lambda}$ and $\frac{\bar{t}}{\lambda}$ (see Lemma 1) are in the interior of the effort space $[0,1]$. Hence, in this case it depends on the previously chosen effort level (and hence the induced value of the agent's outside option) whether the agent decides to stay in both states (for $e \in E_{2}(t)=\left[0, \frac{t}{\lambda}\right]$ ), in the good state $s=\bar{s}$ only (for $e \in E_{1}(t)=\left(\frac{t}{\lambda}, \frac{\bar{t}}{\lambda}\right]$ ), or whether he decides to stay in neither state (for $\left.e \in E_{0}(t)=\left(\frac{\bar{t}}{\lambda}, 1\right]\right)$.

\footnotetext{
${ }^{10}$ Note that we assume that the agent stays when indifferent, which is also the socially efficient choice.
} 
(i)

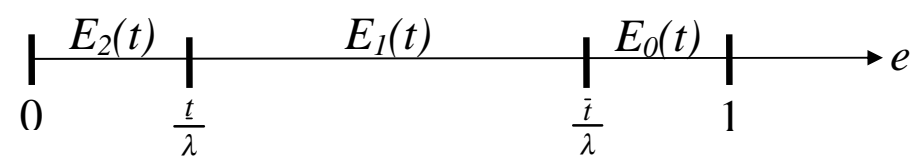

(ii)

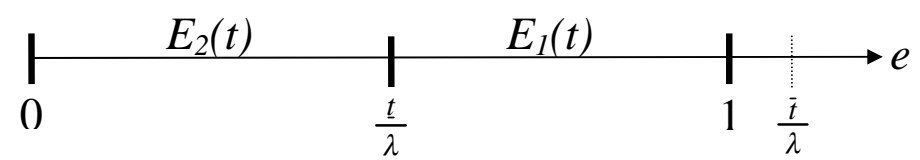

(iii)

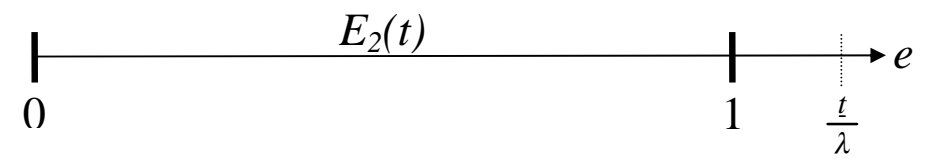

Figure 2: Partition of the effort space induced by a given contract: 3 examples

Second, for contracts such as the one depicted in panel (ii), even when choosing maximum effort (thereby also maximizing his outside option), the agent does not find it optimal to leave in state $s=\bar{s}$. For such contracts, the set $E_{0}(t)$ is empty. However, and similar to panel (i), it still depends on the agent's effort level whether he optimally stays in both states (for $\left.e \in E_{2}(t)=\left[0, \frac{t}{\lambda}\right]\right)$ or in state $s=\bar{s}$ only (for $\left.e \in E_{1}(t)=\left(\frac{t}{\lambda}, 1\right]\right)$. Finally, as for panel (iii), even the payment $\underline{t}$ is sufficiently attractive such that, independent of his outside option, the agent prefers to stay in both states of the world. Here, both $E_{1}(t)$ and $E_{0}(t)$ are empty, and we have $E_{2}(t)=[0,1]$. This leads to the following result (illustrated in Figure 3):

\section{Lemma 2 (Agent's payoff function given optimal ex-post participation decisions)}

(i) For a given contract $t \in T^{+}$and optimal ex-post participation decisions, the agent's expected payoff is given by

$$
U(e, t)= \begin{cases}U_{2}(e, t) \equiv e \cdot \bar{t}+(1-e) \cdot \underline{t}-\frac{1}{2} e^{2} & \text { if } e \in E_{2}(t) \\ U_{1}(e, t) \equiv e \cdot \bar{t}+(1-e) \cdot \lambda e-\frac{1}{2} e^{2} & \text { if } e \in E_{1}(t) \\ U_{0}(e, t) \equiv \lambda e-\frac{1}{2} e^{2} & \text { if } e \in E_{0}(t),\end{cases}
$$

where (a) $E_{2}(t), E_{1}(t), E_{0}(t) \neq \varnothing$ for $\bar{t}<\lambda,{ }^{11}$ (b) $E_{2}(t), E_{1}(t) \neq \varnothing$ and $E_{0}(t)=\varnothing$ for $\underline{t}<\lambda \leq \bar{t}$, and $(c) E_{2}(t) \neq \varnothing$ and $E_{1}(t)=E_{0}(t)=\varnothing$ for $\underline{t} \geq \lambda$,

(ii) $U(e, t)$ is continuous in $e$, and $U_{2}(e, t), U_{1}(e, t)$, and $U_{0}(e, t)$ are strictly concave in $e$.

(iii) $\frac{\partial U_{2}\left(e=\frac{t}{\lambda}, t\right)}{\partial e}<\frac{\partial U_{1}\left(e=\frac{t}{\lambda}, t\right)}{\partial e}$ for $\underline{t}<\lambda$, and $\frac{\partial U_{1}\left(e=\frac{\bar{t}}{\lambda}, t\right)}{\partial e}<\frac{\partial U_{0}\left(e=\frac{\bar{t}}{\lambda}, t\right)}{\partial e}$ for $\bar{t}>0$.

\footnotetext{
${ }^{11}$ For the knife-edge case $\bar{t}=\underline{t}<\lambda$, we have $E_{1}(t)=\varnothing$.
} 


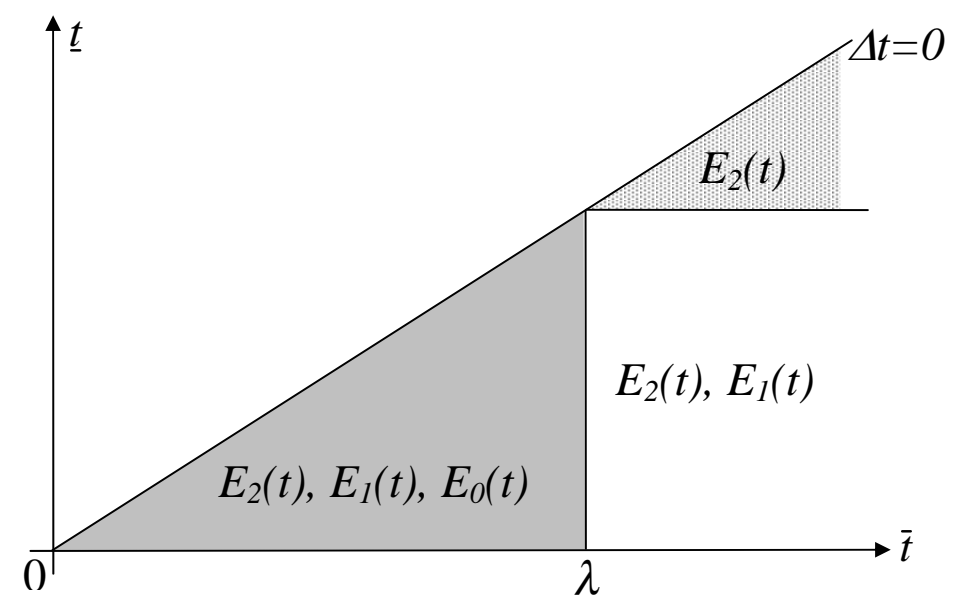

Figure 3: Non-empty sets $E_{j}(t)$ depending on the underlying contract $t$

Step 3: Candidate optimal effort levels Given the payoff function (5), the agent's optimal effort choice $e^{*}(t)$ solves

$$
e^{*}(t) \in \underset{e \in[0,1]}{\arg \max } U(e, t)
$$

Note that solving (6) is non-trivial. First, the chosen effort may affect the agent's subsequent ex-post participation decision. Hence, the agent has to decide from which of the effort intervals $E_{j}(t)$ to select his effort (where, for a given contract, some of these intervals may not exist (see Lemma 2)). Second, as (5) is piece-wise defined, there might, in principle, be a corner solution at one of the threshold values $\frac{t}{\lambda}$ or $\frac{\bar{t}}{\lambda}$. However, one can show that such corner solutions play only a limited role. For an illustration, consider Figure 2 (i), where $\frac{t}{\lambda}, \frac{\bar{t}}{\lambda} \in(0,1)$ holds. Lemma 2 (iii) implies that, at the threshold value $\frac{t}{\lambda}$, the slope of $U(e, t)$ makes a positive jump, because for all $e \in E_{1}(t)$ the agent leaves in the bad state, in which case his payoff $\lambda e$ is increasing in $e$. An analogous argument applies to the boundary between the sets $E_{1}(t)$ and $E_{0}(t)$. Intuitively, these observations imply that choosing $e=\frac{t}{\lambda}$ respectively $e=\frac{\bar{t}}{\lambda}$ cannot be optimal for the agent. In particular, we show below that, with the possible exception of being equal to one, the optimal effort level will satisfy an appropriate first-order condition. To this end, for $j \in\{0,1,2\}$, implicitly define $e_{j}^{*}(t)$ by $\frac{\partial U_{j}(e, t)}{\partial e}=0$, where it follows from (5) that $e_{2}^{*}(t)=\Delta t, e_{1}^{*}(t)=\frac{\bar{t}+\lambda}{2 \lambda+1}$, and $e_{0}^{*}(t)=\lambda .^{12}$

\footnotetext{
${ }^{12}$ Note that $e_{j}^{*}(t)$ does not necessarily lie in $E_{j}(t)$ (or in $[0,1]$ for that matter), but, of course, the subsequent results take these restrictions into account.
} 
Lemma 3 (Candidate optimal effort levels for a given contract) For all $t \in T^{+}$,

(i) if $e^{*}(t)<1$, then, in both states of the world, the agent's equilibrium ex-post participation constraint is not binding, i.e., $e^{*}(t) \neq\left\{\frac{t}{\lambda}, \frac{\bar{t}}{\lambda}\right\}$, and

(ii) there exists some $j \in\{0,1,2\}$ such that $e^{*}(t)=\min \left(e_{j}^{*}(t), 1\right)$ and $\min \left(e_{j}^{*}(t), 1\right) \in E_{j}(t)$.

For any interior effort level, Lemma 3 (i) implies that, if the principal wants the agent to stay in a given state, the agent necessarily reaps a rent in this state. This makes inducing participation more costly compared to the standard model with full commitment, where the agent reaps a rent in some states only.

Step 4: Optimal agent behavior for a given contract Part (ii) of Lemma 3 is useful because, as will be shown below, it leads to a partition of the contract space $T^{+}$into three subsets $T_{0}, T_{1}$, and $T_{2}$ that differ with respect to the induced equilibrium ex-post participation decisions (for a preview, see Figure 4). Intuitively, in the set of full-separation contracts $T_{0}$, the stipulated payments are relatively low in both states such that the agent's outside option becomes relatively attractive. Consequently, the agent will optimally choose a relatively high effort level, and he will optimally stay in neither state. In contrast, in the set of no-separation contracts $T_{2}$, both payments are sufficiently high such that the agent will optimally choose a relatively low effort level (which keeps his outside option low), and he will optimally stay with the principal in both states. Finally, as for the set of partial-separation contracts $T_{1}$, only payment $\bar{t}$ is sufficiently large, which will induce the agent to choose an intermediate effort level such that he will optimally stay with the principal in the high state only.

Before stating the formal result, we define a boundary function $b(\bar{t})$, illustrated in Figure 4, that delineates the sets $T_{0}, T_{1}$, and $T_{2}$ and simplifies their formal characterization in Proposition 1 below. Moreover, this boundary function will play a crucial role when deriving the principal's optimal choice of contract in Section 4. To simplify the subsequent exposition, note that both $U_{0}(e, t)$ and $e_{0}^{*}(t)=\lambda$ are independent of $t$. Consequently, (under slight abuse of notation) define $U_{0} \equiv U_{0}(\min (\lambda, 1), t)$. 
Definition 1 (Boundary function) Define a (continuous) function $b: \bar{t} \rightarrow \underline{t}$ as follows:

(i) for $\bar{t} \in\left[\bar{t}^{\prime}, \bar{t}^{\prime \prime}\right]: \quad b(\bar{t})$ is implicitly defined by $U_{2}\left(e_{2}^{*}(t), t\right)=U_{0}$

(ii) for $\bar{t} \in\left[\bar{t}^{\prime \prime}, \bar{t}^{\prime \prime \prime}\right]: \quad b(\bar{t})$ is implicitly defined by $U_{2}\left(e_{2}^{*}(t), t\right)=U_{1}\left(e_{1}^{*}(t), t\right)$, and

(iii) for $\bar{t} \in\left[t^{\prime \prime \prime}, \infty\right): \quad b(\bar{t})=\lambda$

where $\bar{t}^{\prime} \equiv U_{0}, \bar{t}^{\prime \prime}$ is implicitly defined by $U_{1}\left(e_{1}^{*}(t), t\right)=U_{0}$, and $\bar{t}^{\prime \prime \prime} \equiv 1+\lambda$, and where $0<\bar{t}^{\prime}<\bar{t}^{\prime \prime}<\bar{t}^{\prime \prime \prime}$ holds.

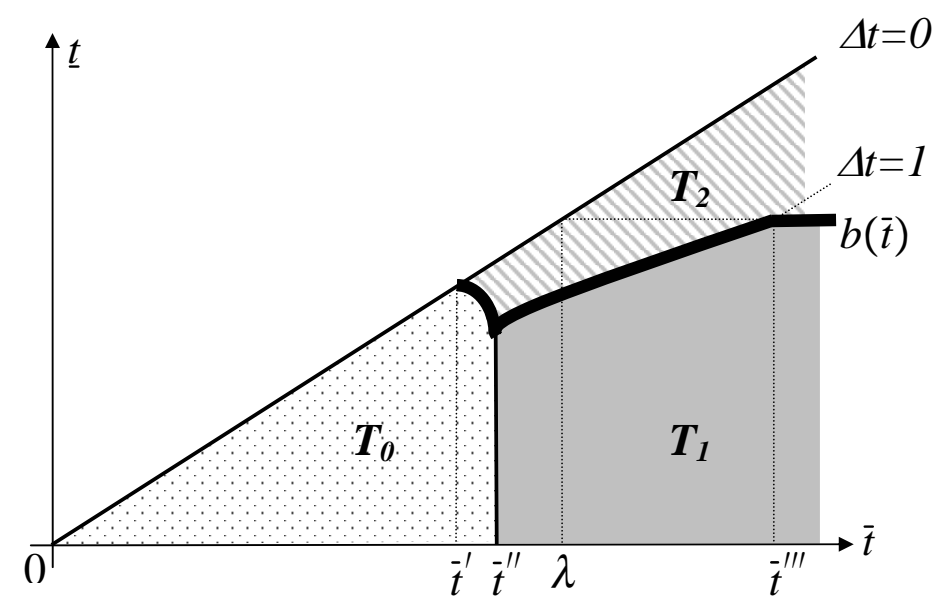

Figure 4: Sets of full-, partial-, and no-separation contracts

This section's main result can now be stated as follows:

Proposition 1 (Optimal agent behavior for a given contract) The contract space $T^{+}$ can be partitioned into three (non-empty) subsets $T_{0}, T_{1}$, and $T_{2}$ that differ in the equilibrium ex-post participation decision of the agent. In particular, for a given contract $t \in T_{j}$, $j \in\{0,1,2\}$, the agent selects equilibrium effort $e^{*}(t)=\min \left(e_{j}^{*}(t), 1\right)$ and subsequently chooses to stay with the principal in $j$ states of the world. Formally, the sets $T_{0}, T_{1}$, and $T_{2}$ are defined by $T_{2} \equiv\left\{t \in T^{+} \mid \underline{t} \geq b(\bar{t})\right.$ and $\left.\bar{t} \geq \bar{t}^{\prime}\right\}, T_{1} \equiv\left\{t \in T^{+} \mid \underline{t}<b(\bar{t})\right.$ and $\left.\bar{t} \geq \bar{t}^{\prime \prime}\right\}$, and $T_{0} \equiv T^{+} \backslash$ $\left(T_{2} \cup T_{1}\right)$, where the continuous boundary function $b(\bar{t})$ is decreasing and concave for $\bar{t} \in\left[\bar{t}^{\prime}, \bar{t}^{\prime \prime}\right]$, and increasing and concave with a slope of less than 1 for $\bar{t} \in\left[\bar{t}^{\prime \prime}, \bar{t}^{\prime \prime \prime}\right]$, and where $b\left(\bar{t}^{\prime}\right)=\bar{t}^{\prime}$. 
As for the optimal effort level $e^{*}(t)$, it follows from Proposition 1 that

$$
e^{*}(t)= \begin{cases}e_{2}^{*}(t) & \text { if } t \in T_{2} \text { and } \Delta t \leq 1, \\ e_{1}^{*}(t) & \text { if } t \in T_{1} \text { and } \bar{t} \leq \bar{t}^{\prime \prime \prime} \\ \min (\lambda, 1) & \text { if } t \in T_{0}, \text { and } \\ 1 & \text { otherwise }\end{cases}
$$

As the boundary function $b(\bar{t})$ will be crucial throughout, some more intuition regarding its shape is useful. ${ }^{13}$ First, to see why it is decreasing in the interval $\left[t^{\prime}, \bar{t}^{\prime \prime}\right]$, recall from Definition 1 that on this part of the graph of $b(\bar{t})$ it has to hold that $U_{2}\left(e_{2}^{*}(t), t\right)=U_{0}$. Note that $U_{0}$ is constant in the $(\underline{t}, \bar{t})$-space, and, from (5) and the Envelope-Theorem, it follows that $\frac{\partial U_{2}\left(e_{2}^{*}(t), t\right)}{\partial \bar{t}}=e_{2}^{*}(t) \geq 0$ and $\frac{\partial U_{2}\left(e_{2}^{*}(t), t\right)}{\partial \underline{t}}=1-e_{2}^{*}(t)>0$. Hence, a lower $\underline{t}$ is required to keep the agent indifferent when raising $\bar{t}$, and vice versa. Second, in the interval $\left[\bar{t}^{\prime \prime}, \bar{t}^{\prime \prime \prime}\right], b(\bar{t})$ is implicitly defined by $U_{2}\left(e_{2}^{*}(t), t\right)=U_{1}\left(e_{1}^{*}(t), t\right)$. Note that $\frac{\partial U_{1}\left(e_{1}^{*}(t), t\right)}{\partial \bar{t}}=e_{1}^{*}(t)$, which again follows from (5) and the Envelope-Theorem. Moreover, the boundary between the sets $T_{2}$ and $T_{1}$ is located in a part of the contract space where $e_{2}^{*}(t)<e_{1}^{*}(t)$ holds (see the proof of Proposition 1). Consequently, a higher $\bar{t}$ raises $U_{1}\left(e_{1}^{*}(t), t\right)$ by more than it raises $U_{2}\left(e_{2}^{*}(t), t\right)$. Hence, to keep the agent indifferent as $\bar{t}$ increases, $\underline{t}$ must go up as well because it affects $U_{2}\left(e_{2}^{*}(t), t\right)$, but not $U_{1}\left(e_{1}^{*}(t), t\right)$ (see $\left.(5)\right)$. Finally, note that since neither $U_{0}$ nor $U_{1}\left(e_{1}^{*}(t), t\right)$ depend on $\underline{t}$, the boundary between the regions $T_{0}$ and $T_{1}$ is a vertical line at $\bar{t}=\bar{t}^{\prime \prime}$.

\section{Optimal contracts}

Taking into account the agent's optimal behavior for a given contract (as characterized in Proposition 1), an optimal contract maximizes the principal's expected payoff (see (1))

$$
\Pi(t) \equiv e^{*}(t) \cdot(\bar{s}-\bar{t}) \cdot \bar{I}+\left(1-e^{*}(t)\right) \cdot(\underline{s}-\underline{t}) \cdot \underline{I}
$$

where $\bar{I}(\underline{I})$ is equal to one iff $\bar{t} \geq \lambda e^{*}(t)\left(\underline{t} \geq \lambda e^{*}(t)\right)$, and zero otherwise. It also follows from Proposition 1 that the principal faces a choice between three types of contracts (i.e., contracts from the sets $T_{2}, T_{1}$, and $T_{0}$, respectively) that differ in the induced effort and ex-post

\footnotetext{
${ }^{13}$ Note that, given a contract on the part of the graph of $b(\bar{t})$ where $\bar{t} \in\left[t^{\prime}, \bar{t}^{\prime \prime}\right]$ holds, the agent is indifferent between the effort level $e_{2}^{*}(t) \in E_{2}(t)$ and the effort level $\min \left(e_{0}^{*}(t), 1\right) \in E_{0}(t)$. However, it will become clear in Section 4 on optimal contracts that, on the equilibrium path, these indifferences are resolved in favor of $e_{2}^{*}(t)$ (which also constitutes the socially efficient behavior). This is already taken into account in the definition of the set $T_{2}$ that is assumed to include the respective part of the graph of $b(\bar{t})$. Similar considerations apply when resolving indifferences in the definitions of $T_{2}, T_{1}$, and $T_{0}$.
} 
participation decisions; thereby leading to either no-, partial-, or full-separation. However, note that the principal's payoff in the latter case is zero, while it will turn out that she can always secure a strictly positive payoff by instead choosing an appropriate no-separation or partialseparation contract. ${ }^{14}$ Consequently, the search for the optimal contract can be restricted to the former two contract types. Hence, we first characterize optimal no-separation and partialseparation contracts, respectively (Propositions 2 and 3). We then compare these two contract types to determine the "globally" optimal contract ultimately chosen by the principal. Thereby, depending on parameters, either contract type can be strictly optimal (Proposition 4).

The optimal no-separation contract When the principal offers a no-separation contract $t \in T_{2}$, then from Proposition 1 , the agent chooses effort $e^{*}(t)=\min (\Delta t, 1)$ and (optimally) stays in both states of the world. It turns out that, in her search for the optimal no-separation contract, the principal can narrow down the set of candidate optimal contracts considerably to include only the upper bold line of Figure 5, which corresponds to the graph of the boundary function $b(\bar{t})$ over the interval $\left[\bar{t}^{\prime}, \bar{t}^{\prime \prime \prime}\right]$.

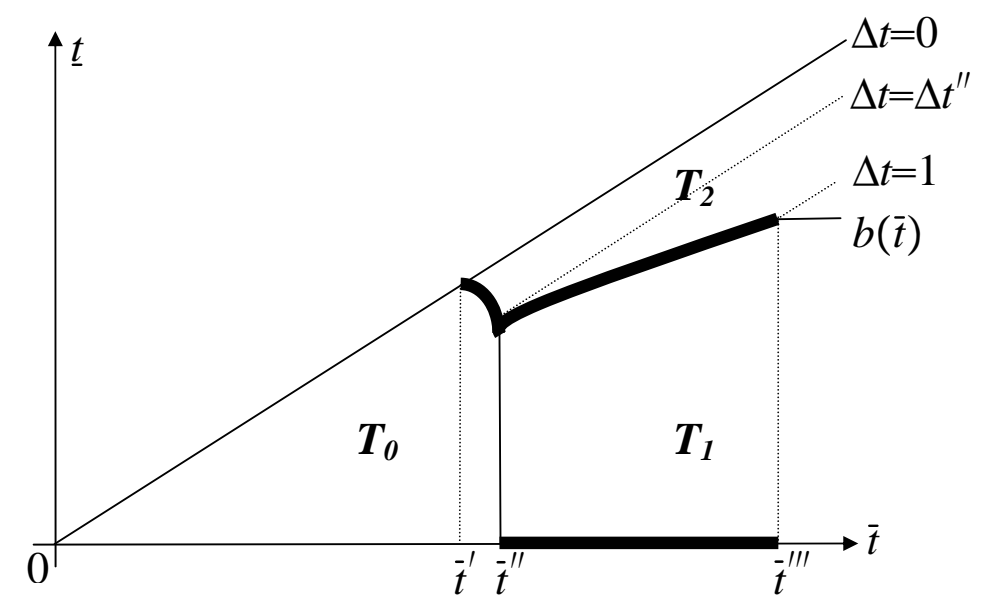

Figure 5: Candidate optimal contracts

Intuitively, as $e^{*}(t)=\min (\Delta t, 1)$, it cannot be optimal for the principal to choose $\Delta t>1$, because there would exist a contract with $\Delta t=1$ that would induce the same effort at lower cost. Moreover, for any given effort $\Delta t$ the principal optimally sets $\underline{t}$ as low as possible. In the standard model with full commitment, this leads to $\underline{t}=0$ which, however, in our setup would

\footnotetext{
${ }^{14}$ This is shown formally in the proof of Proposition 4.
} 
induce the agent to leave for $s=\underline{s}$. Rather, to implement a given effort level $\Delta t$, the respective payment $\underline{t}$ must be sufficiently high to avoid separation. Consider the upper bold line of Figure 5 and note that effort levels $\Delta t$ can be depicted as lines parallel to the main diagonal. Hence, the cost-minimizing payment $\underline{t}$ is given by the left-most point on the respective parallel line that is still inside $T_{2}$, i.e., the optimal contract implementing a given $\Delta t$ lies in the (unique) point of intersection of the respective parallel with $b(\bar{t}) .{ }^{15}$

In analogy to the standard model, the optimal no-separation contract exhibits the natural feature that the effort level $e=\Delta t$ optimally implemented by the principal does not depend on the absolute levels $\bar{s}$ and $\underline{s}$, but depends on the spread $\Delta s$ only, and is (weakly) increasing in $\Delta s$. However, the principal's need to set both payments sufficiently high (to avoid separation ex-post) leads to properties of optimal no-separation contracts markedly different from the standard model:

Proposition 2 (Optimal no-separation contract) For given $(\underline{s}, \bar{s})$, the optimal contract in the set $T_{2}$ has the following properties:

(i) To induce some effort level $e=\Delta t \leq 1$, it is given by $t=(b(\bar{t}), \bar{t})$ satisfying $\bar{t}-b(\bar{t})=\Delta t$.

(ii) The effort level $e=\Delta t$ optimally induced by the principal depends on $\Delta s$ only, and is continuous and weakly increasing in $\Delta s$ (see Figure 6). Formally,

$$
\begin{gathered}
\quad \Delta t=\left\{\begin{array}{lll}
\Delta s & =e^{F B}(\Delta s) & \text { if } \Delta s \in\left(0, \Delta t^{\prime \prime}\right], \\
\Delta t^{\prime \prime} & =\text { const. }<e^{F B}(\Delta s) & \text { if } \Delta s \in\left(\Delta t^{\prime \prime}, \Delta s_{I}\right], \\
\frac{\Delta s}{2}-\frac{\sqrt{1+2 \lambda}-1}{2}<e^{F B}(\Delta s) & \text { if } \Delta s \in\left(\Delta s_{I}, \Delta s_{I I}\right], \\
1 & \text { if } \Delta s>\Delta s_{I I},
\end{array}\right. \\
\text { where } 0<\Delta t^{\prime \prime} \equiv \bar{t}^{\prime \prime}-b\left(\bar{t}^{\prime \prime}\right)<\Delta s_{I} \equiv 2 \Delta t^{\prime \prime}+\sqrt{1+2 \lambda}-1<\Delta s_{I I} \equiv 1+\sqrt{1+2 \lambda} .
\end{gathered}
$$

In our view, the most interesting property of the optimal no-separation contract is that the additional source of frictions (limited commitment in addition to moral hazard and limited liability) does not necessarily lead to higher agency costs in equilibrium. To the contrary, it might turn out to be welfare-improving, and even first-best effort incentives (and hence, first-

\footnotetext{
${ }^{15}$ Proposition 1 implies that there is a unique point of intersection because $\bar{t}^{\prime}-b\left(\bar{t}^{\prime}\right)=0, \bar{t}-b(\bar{t})$ is strictly increasing in $\bar{t}$ for $\bar{t} \in\left[\bar{t}^{\prime}, \bar{t}^{\prime \prime \prime}\right]$, and $\bar{t}^{\prime \prime \prime}-b\left(\bar{t}^{\prime \prime \prime}\right)=1$.
} 


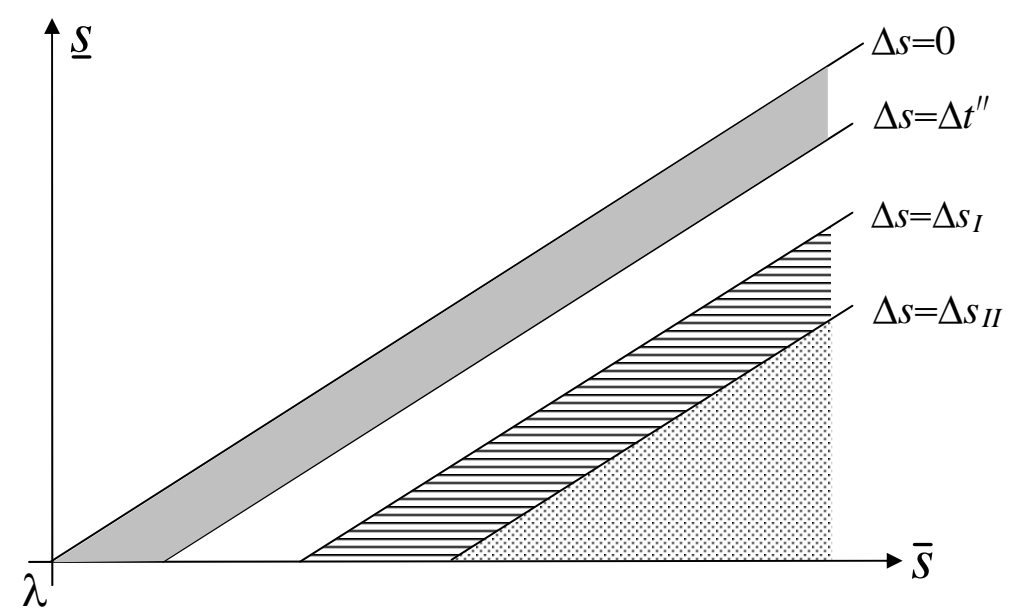

Figure 6: Regions of optimal no-separation contracts

best surplus) might be induced by the principal. ${ }^{16}$ Moreover, as shown in Proposition 4 below, whenever $\Delta s \leq \Delta t^{\prime \prime}$ the optimal no-separation contract not only leads to first-best incentives but is also "globally" optimal for the principal.

To gain an intuition, note that the discussion above Proposition 2 implies that the principal's problem of maximizing (8) can be transformed into the one-dimensional problem of finding the optimal $\bar{t} \in\left[\bar{t}^{\prime}, \bar{t}^{\prime \prime \prime}\right]$. This is so because each point on the graph of $b(\bar{t}$ ) (and hence, each effort level $e=\Delta t$ ) is identified by a unique value of $\bar{t}$. Moreover, it follows from Proposition 1 and (8) that for all $t \in T_{2}$ that satisfy $\Delta t \leq 1$, we have $\Pi(t)=W(t)-U_{2}\left(e_{2}^{*}(t), t\right)$, where $W(t)$ denotes the social surplus as a function of $t$, i.e.,

$$
W(t) \equiv \Pi(t)+U\left(e^{*}(t), t\right) \text { for } t \in T^{+} .
$$

These observations in combination with Definition 1 imply that the principal's problem can be stated as finding the $\bar{t} \in\left[\bar{t}^{\prime}, \bar{t}^{\prime \prime \prime}\right]$ that maximizes

$$
\Pi(t)=W(t)- \begin{cases}U_{0} & \text { if } \bar{t} \in\left[\bar{t}^{\prime}, \bar{t}^{\prime \prime}\right] \\ U_{1}\left(e_{1}^{*}(t), t\right) & \text { if } \bar{t} \in\left[\bar{t}^{\prime \prime}, \bar{t}^{\prime \prime \prime}\right]\end{cases}
$$

under the constraint that $\underline{t}=b(\bar{t})$.

The intuition behind Proposition 2 then follows naturally from the properties of (10) (see also Figure 6 for an illustration). First, as long as $\Delta s$ is not too large, the principal finds it

\footnotetext{
${ }^{16}$ At the same time, the principal's equilibrium payoff is lower than in the standard model with full commitment, because the optimal no-separation contract has to satisfy the additional constraints of inducing participation ex-post.
} 
optimal to implement a relatively small effort level, and she achieves this by choosing some $\bar{t}<\bar{t}^{\prime \prime}$. It follows from (10) that, in this range, her payoff is equal to the social surplus minus a constant; making her residual claimant and implying the optimality of first-best incentives.

Second, there exists an intermediate range of $\Delta s$ for which the optimal $\bar{t}$ (and hence, the induced effort level) is constant in $\Delta s$ (and below first-best effort). Intuitively, note from (10) that for $\bar{t} \geq \bar{t}^{\prime \prime}$, the agent's payoff is given by $U_{1}\left(e_{1}^{*}(t), t\right)$. Moreover, recall that $U_{1}\left(e_{1}^{*}(t), t\right)$ depends on $\bar{t}$ only and $\frac{\partial U_{1}\left(e_{1}^{*}(t), t\right)}{\partial \bar{t}}=e_{1}^{*}(t)>0$ for $\bar{t} \in\left[\bar{t}^{\prime \prime}, \bar{t}^{\prime \prime \prime}\right]$. Hence, while for $\bar{t}<\bar{t}^{\prime \prime}$ the agent's marginal rent is zero, it jumps to $e_{1}^{*}(t)$ at $\bar{t}=\bar{t}^{\prime \prime}$. Due to this jump in the marginal rent, the principal's benefit from raising $\bar{t}$ must be sufficiently large to make it worthwhile to raise $\bar{t}$ beyond $\bar{t}^{\prime \prime}$, explaining why for an entire range of $\Delta s$ the optimal $\bar{t}$ remains constant at $\bar{t}^{\prime \prime}$.

Finally, as $\Delta s$ increases further, by choosing some $\bar{t} \in\left(\bar{t}^{\prime \prime}, \bar{t}^{\prime \prime \prime}\right)$, the principal optimally induces some effort level that is again strictly increasing in $\Delta s$. In this case, the agent's marginal rent $e_{1}^{*}(t)$ is larger (and hence, the implemented effort is lower) than in the standard model with full commitment, where the induced effort is given by $\min \left(\frac{\Delta s}{2}, 1\right)$ (see Proposition 5). The reason is that, to induce a higher effort level and to avoid separation, the principal has to raise both $\underline{t}$ and $\bar{t}$, which is in contrast to the standard model where a higher effort is induced by raising the payment in the good state only.

The optimal partial-separation contract As shown in Proposition 1, when offered a partial-separation contract $t \in T_{1}$, the agent exerts effort $e^{*}(t)=\min \left(\frac{\bar{t}+\lambda}{2 \lambda+1}, 1\right)$ and optimally stays in state $s=\bar{s}$ only. In a first step, we argue that in the set $T_{1}$, without loss of generality, one can restrict attention to contracts where $\underline{t}=0$ (i.e., those located on the lower bold line of Figure 5): the agent leaves if state $\underline{s}$ is realized, which implies that $\Pi(t)$ does not depend on $\underline{t}$. Moreover, payments $\bar{t}>\bar{t}^{\prime \prime \prime}$ cannot be optimal for the principal as they would induce the same effort level of 1 as a contract stipulating $\bar{t}=\bar{t}^{\prime \prime \prime}$, but at a higher cost. These observations imply that (in order to find the optimal partial-separation contract) the principal's problem of maximizing (8) can be restated as finding the $\bar{t} \in\left[\bar{t}^{\prime \prime}, \bar{t}^{\prime \prime \prime}\right]$ that maximizes

$$
\Pi(t)=W(t)-U_{1}\left(e_{1}^{*}(t), t\right)=\left(\frac{\bar{t}+\lambda}{1+2 \lambda}\right) \cdot(\bar{s}-\bar{t}) .
$$

As $\Pi(t)$ is independent of $\underline{s}$, compared to the no-separation case, the characterization of the optimal partial-separation contract is substantially less complex: 
Proposition 3 (Optimal partial-separation contract) For given $(\underline{s}, \bar{s})$, the optimal contract in the set $T_{1}$ has the following properties:

(i) The payment $\underline{t}$ is irrelevant, and, without loss of generality, some contract of the form $\underline{t}=0$ and $\bar{t} \in\left[t^{\prime \prime}, \bar{t}^{\prime \prime \prime}\right]$ is optimal.

(ii) The effort optimally induced by the principal and the required payment $\bar{t}$ are independent of $\underline{s}$, and continuous and weakly increasing in $\bar{s}$ (see Figure 7). In particular,

$$
\bar{t}=\left\{\begin{array}{lll}
\bar{t}^{\prime \prime} & \text { if } \bar{s} \in\left(0, s_{I}\right], & \text { implying } e=\frac{\bar{t}^{\prime \prime}+\lambda}{2 \lambda+1} \\
\frac{\bar{s}-\lambda}{2} & \text { if } \bar{s} \in\left(s_{I}, s_{I I}\right], & \text { implying } e=\frac{\bar{s}+\lambda}{4 \lambda+2} \\
\bar{t}^{\prime \prime \prime} & \text { if } \bar{s} \in\left(s_{I I}, \infty\right), & \text { implying } e=1
\end{array}\right.
$$

where $s_{I} \equiv 2 \bar{t}^{\prime \prime}+\lambda<s_{I I} \equiv 2 \bar{t}^{\prime \prime \prime}+\lambda$.

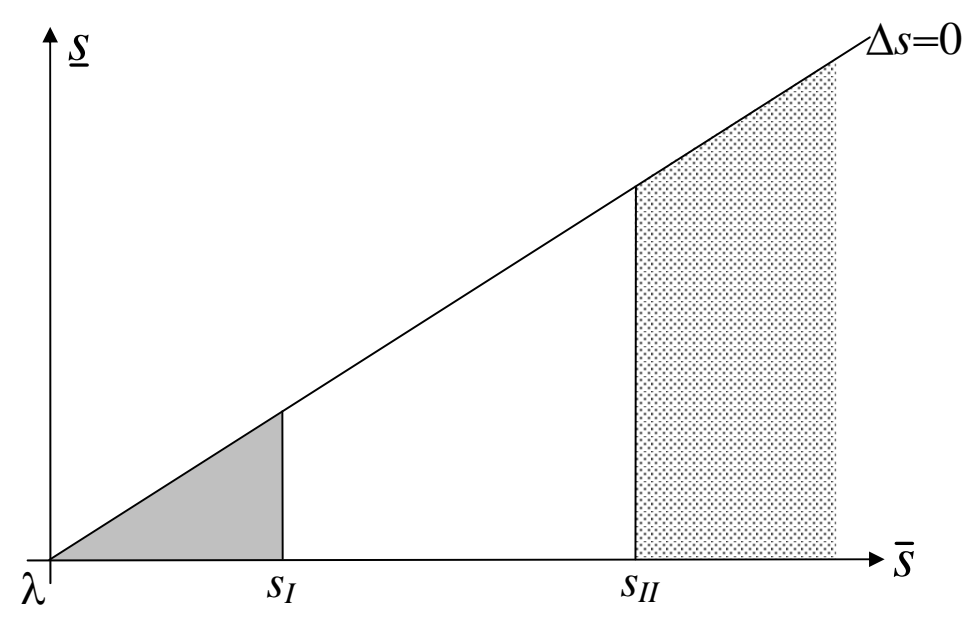

Figure 7: Regions of optimal partial-separation contracts

The optimal partial-separation contract has a number of interesting properties. First, for $s=\underline{s}$ it induces separation, which is socially inefficient because our assumption $\lambda<\underline{s}$ implies $e^{*}(t) \lambda<\underline{s}$. Second, separation in the bad state is induced through a sufficiently unattractive payment $\underline{t}=0$ such that the agent prefers to leave. This feature is reminiscent of up-or-out policies, which (similar to the present case) are often enforced informally (e.g., by offering an employee a very low bonus, by moving him to a less intriguing job, or by making clear his lacking career options); inducing the employee to leave voluntarily. ${ }^{17}$

\footnotetext{
${ }^{17}$ See Walker (2005) for empirical evidence on implicit up-or-out policies in a large manufacturing firm.
} 
Third, the agent may in fact be harmed by a more attractive outside option, i.e., the agent's equilibrium payoff may be decreasing in $\lambda^{18}$ To see this, denote the optimal partialseparation contract by $t_{1}=\left(0, \bar{t}_{1}\right)$ where $\bar{t}_{1}$ denotes the optimal payment in state $\bar{s}$ as identified in Proposition 3. Under this contract, the derivative of the agent's expected payoff with respect to $\lambda$ is given by

$$
e_{1}^{*}\left(t_{1}\right) \cdot \frac{\partial \bar{t}_{1}}{\partial \lambda}+\left(1-e_{1}^{*}\left(t_{1}\right)\right) \cdot e_{1}^{*}\left(t_{1}\right)
$$

which follows from (5), Proposition 1, and the Envelope-Theorem. ${ }^{19}$ In the following, suppose $\bar{s}<s_{I I}$ such that the induced effort level is interior (see Proposition 3). The second term in (12) is a direct, positive effect: a larger $\lambda$ implies a larger payoff for the agent in the state where he leaves. The first term denotes an indirect effect that turns out to be negative. Intuitively, as long as the induced effort level is interior, $\bar{t}_{1}$ and $\lambda$ (as internal respectively external sources of effort incentives) are substitutes. That is, a larger $\lambda$ reduces the positive effect of $\bar{t}_{1}$ on effort. Hence, the principal optimally responds to a larger $\lambda$ by reducing $\bar{t}_{1}$. It turns out that, for $\bar{s} \in\left(s_{I}, s_{I I}\right)$ and $\lambda>1$, this negative, indirect effect in fact dominates the positive direct effect, and the agent's expected payoff is decreasing in $\lambda .{ }^{20}$

The globally optimal contract It remains to determine which of the two candidate optimal contracts (as characterized in Propositions 2 and 3) the principal ultimately prefers. It turns out that either contract type may be strictly optimal. In particular, there always exists a parameter region $(\underline{s}, \bar{s})$ where a partial-separation contract is optimal for the principal. To see this, consider Figure 8 (which combines Figures 6 and 7) and the following "revealed preference" argument. Recall from Proposition 2 that for all $\Delta s \geq \Delta s_{I I}$, the principal implements effort $e=1$ under the optimal no-separation contract. Moreover, if $\bar{s} \geq s_{I I}$ holds in addition, then $e=1$ is also implemented under the optimal partial-separation contract (see Proposition 3). In this case, both contracts are payoff-equivalent, and the principal is indifferent between the two contracts. Now, consider the white, dotted area in Figure 8, which satisfies $\bar{s}<s_{I I}$.

\footnotetext{
${ }^{18}$ Wickelgren (2009) shows that an improved outside option can worsen a player's payoff in a bargaining game with strategic delay.

${ }^{19}$ Note that both the optimal partial-separation contract $t_{1}$ and the effort level $e^{*}\left(t_{1}\right)$ directly depend on $\lambda$ (see Proposition 3 and the discussion above Lemma 3), which is taken into account when obtaining the derivative.

${ }^{20}$ Formally, in this parameter range, $e_{1}^{*}\left(t_{1}\right)=\frac{\bar{t}_{1}+\lambda}{2 \lambda+1}$, and hence the agent's expected payoff is $\frac{(\bar{s}+\lambda)^{2}}{8(1+2 \lambda)}$, which is strictly decreasing in $\lambda$ iff $\bar{s}>1+\lambda$. At the same time, it can be shown that $s_{I} \equiv 2 \bar{t}^{\prime \prime}+\lambda=2 \sqrt{4 \lambda^{2}-1}-\lambda>1+\lambda$, which implies the result.
} 


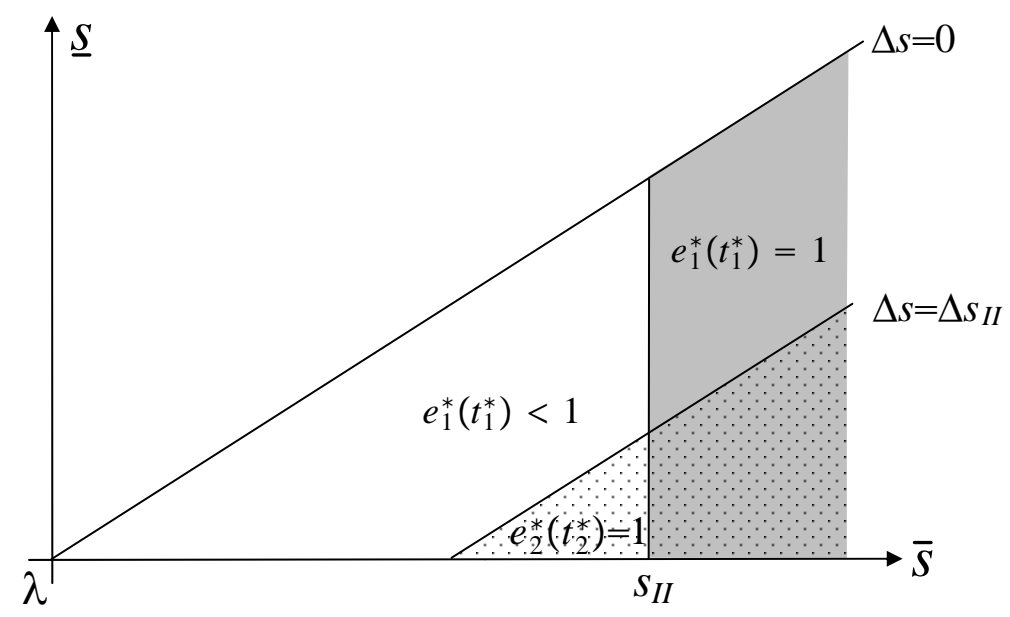

Figure 8: Global optimality of partial-separation: a revealed-preference argument

There, the induced effort level under the optimal partial-separation contract is less than 1, even though implementing $e=1$ would have been feasible. Hence, by optimality, it follows that the principal must strictly prefer this partial-separation contract to the optimal no-separation contract which, recall, is payoff-equivalent to the partial-separation contract that implements $e=1$. Strict optimality of partial-separation contracts does, however, not depend on the existence of corner solutions as the following result shows.

Proposition 4 (Globally optimal contract) There is a strictly increasing, continuous function $B: \underline{s} \rightarrow \bar{s}$ that allows to partition the $(\underline{s}, \bar{s})$-space into three subsets (see Figure 9):

(i) For $\bar{s}<B(\underline{s})$, the no-separation contract (Proposition 2) is strictly optimal. In particular, this is the case when $\Delta s \leq \Delta t^{\prime \prime}$ holds. Moreover, for all $\Delta s \in\left(\Delta t^{\prime \prime}, \Delta s_{I I}\right)$ there exists a range of values of $\underline{s}$ and $\bar{s}$ such that no-separation is strictly optimal.

(ii) For $B(\underline{s})<\bar{s}<s_{I I}$, the partial-separation contract (Proposition 3) is strictly optimal,

(iii) Otherwise, the principal is indifferent between these two contracts. In particular, this is the case when both $\bar{s} \geq s_{I I}$ and $\Delta s \geq \Delta s_{I I}$ hold.

First, for $\Delta s \leq \Delta t^{\prime \prime}$ the no-separation contract is globally optimal because in this parameter range, the principal's payoff is given by the first-best social surplus minus the constant $U_{0}$ (see (10) and Proposition 2). At the same time, from Proposition 3, the optimal partial-separation contract has the form $\left(\underline{t}=0, \bar{t} \in\left[\bar{t}^{\prime \prime}, \bar{t}^{\prime \prime \prime}\right]\right)$ in which case, the principal's payoff is given by 


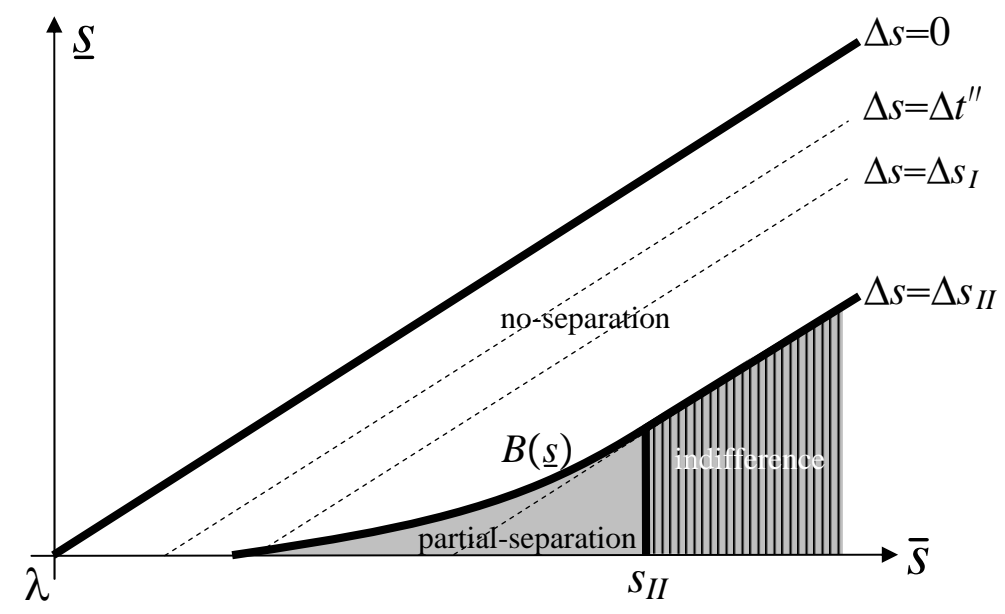

Figure 9: Globally optimal contracts

$W(t)-U_{1}\left(e_{1}^{*}(t), t\right)$ (see (11)), where $W(t)$ is below the first-best social surplus and where $U_{1}\left(e_{1}^{*}(t), t\right) \geq U_{0}$ for all $\bar{t} \in\left[\bar{t}^{\prime \prime}, \bar{t}^{\prime \prime \prime}\right] .{ }^{21}$

Second, while no-separation is strictly optimal for $\Delta s$ not too large, it becomes less attractive for the principal as $\Delta s$ (and hence the implemented effort) increases. This is due to the fact that, at the same time, the agent's rent becomes larger. As a result, for $\Delta s$ sufficiently large, partial-separation becomes optimal for the principal: while foregoing some of the surplus, this allows the principal to save on the agent's rent and to free-ride on the (external) effort incentives generated by the agent's endogenous outside option.

\section{Discussion}

In this section, first, we briefly consider the potential role (and desirability) of non-compete clauses in restricting the agent's freedom to leave. Second, to illustrate that our results are driven by the combination of the agent's freedom to leave and the endogeneity of his outside option, we also analyze the case where his ex-post outside option is independent of his effort, and hence exogenous. Third, we show that the restriction to the class of contracts introduced in section 2 is without loss of generality. Finally, we relax the assumption that the principal can commit to the initial contract and allow for renegotiation.

\footnotetext{
${ }^{21}$ From Definition 1 recall that for $\bar{t}=\bar{t}^{\prime \prime}$, we have $U_{0}=U_{1}\left(e_{1}^{*}(t), t\right)$, and $\frac{\partial U_{1}\left(e_{1}^{*}(t), t\right)}{\partial \bar{t}}=e_{1}^{*}(t)>0$ for $\bar{t} \in\left[\bar{t}^{\prime \prime}, \bar{t}^{\prime \prime \prime}\right]$.
} 
Freedom to leave and non-compete clauses In the model, the agent is free to leave to pursue his ex-post outside option. In principle, the principal might try to prevent this through instruments such as non-compete clauses as can be observed, for example, in the context of employment relationships. However, in practice, courts are often hesitant to enforce non-compete clauses; worrying that employers might abuse them to prevent employees from working elsewhere at all. As a result, courts typically allow employees to leave except for cases where the current employer's legitimate business interests (e.g., trade secrets) are affected. ${ }^{22}$ One extreme case in this respect is California, where non-compete clauses are not enforced at all. ${ }^{23}$ Such legal practices do find support in our framework in those cases where the agent's freedom to leave leads to the first-best surplus.

Freedom to leave and exogenous outside option Now, suppose that the agent's ex-post outside option is given by $v(e)=\lambda$ (and hence independent of $e$ ), where, in analogy to above, $0 \leq \lambda<\underline{s}$. It turns out that in this setting, the resulting effort level and ex-post participation decisions are identical to those observed in the standard model with full commitment (see e.g., Laffont and Martimort, 2002, p. 194ff.). To see this, note that in the bad (good) state the agent will leave the principal ex-post if $\underline{t}<\lambda(\bar{t}<\lambda)$ holds. Now, suppose that the optimal contract would indeed stipulate $\underline{t}<\lambda$, in which case the agent would leave, and the payoffs of the agent and principal would be $\lambda$ and 0 , respectively. However, in this case the principal would be better off by setting $\underline{t}=\lambda$, while leaving $\bar{t}$ unchanged: the agent would then face the same effort incentives and would obtain the same payoff as before. However, the principal would be strictly better off, because the agent would now stay in the bad state (maintaining our assumption that he does so when indifferent); yielding the principal an extra payoff of $\underline{s}-\lambda>0$ in this state. It follows that under the optimal contract, $\underline{t} \geq \lambda$ and (by an analogous argument) $\bar{t} \geq \lambda$ have to hold. Put differently (and in contrast to the framework analyzed so

\footnotetext{
${ }^{22}$ A prominent example is Mark Papermaster, who (despite of a non-compete clause) moved from IBM to Apple Inc. (to replace Tony Fadell, "father" of the iPod, as Senior Vice President). While IBM tried to stop the move, the case was finally settled in 2009: Papermaster was allowed to move with the only condition of having to testify in court that he would not share IBM business secrets (see e.g., http://www.ft.com/cms/s/0/111b3c1caadd-11dd-897c-000077b07658.html and http://www.apple.com/pr/library/2009/01/27papermaster.html).

${ }^{23}$ For more detailed discussions of non-compete clauses, see e.g., Posner, Triantis, and Triantis (2004) and Gilson (1999), who, however, do not focus on effort incentives (for an exception, see e.g., Kräkel and Sliwka, 2009). The issue of optimal litigation strategies against leaving knowledge workers is analyzed in Gompers and Lerner (2000) and Hvide and Kristiansen (2009).
} 
far), the optimal contract is always of the no-separation type. Hence, in line with the standard model, the agent's optimal choice of effort level is given by the wage spread $\Delta t$, and the wage spread optimally chosen by the principal depends on $\Delta s$ only. However, as for the respective payments, the principal cannot set $\underline{t}$ as low as possible, but has to respect the agent's ex-post outside option which leads to $\underline{t}=\lambda$. In contrast to the endogenous outside option case, this implies that the agent does not earn a rent in state $\underline{s}$ (i.e., he is indifferent between staying and leaving); making no-separation more attractive in the exogenous outside option case. ${ }^{24}$

Proposition 5 (Optimal contract with exogenous outside option) If (i) the agent is free to leave and (ii) his ex-post outside option is exogenous and given by $v(e)=\lambda$, where $0 \leq \lambda<\underline{s}$, then the optimal contract is of the no-separation type and satisfies $\underline{t}=\lambda$ and $\Delta t=\min \left(\frac{\Delta s}{2}, 1\right)$. The induced effort level is $e=\min \left(\frac{\Delta s}{2}, 1\right)$. Moreover, for $\lambda=0$, the equilibrium outcome is exactly the same as in the standard model with full commitment.

Note that, for $\lambda>0$, compared to the standard model, only the optimal payments go up by $\lambda$ in both states of the world. Hence, the main predictions of the standard model are qualitatively unaffected by the agent's freedom to leave as long as his outside option is exogenous (i.e., as long as the agent's effort is fully specific to the relationship with the principal, and hence does not affect the value of his outside option). In contrast, as shown above, the agent's freedom to leave leads to markedly different results if his outside option is endogenous (i.e., if the agent's effort has at least some general component that positively affects his outside option).

Contract space Up to now, we have only considered contracts of the form $t \in T^{+} \equiv\{(\underline{t}, \bar{t}) \mid$ $\bar{t} \geq \underline{t} \geq 0\}$ and have argued that this restriction is without loss of generality. We now prove this claim formally. The revelation principle implies that it suffices to consider a set $T$ of contracts $\left(\tau(\widehat{e}, \widehat{s} ; s), \tau_{0}(\widehat{e}, \widehat{s})\right)$, where (i) $\widehat{e}$ and $\widehat{s}$ denote messages about $e$ and $s$, respectively, that the agent sends after date 3 , (ii) for given messages $\widehat{e}$ and $\widehat{s}, \tau(\widehat{e}, \widehat{s} ; s)$ denotes the payment to the agent if the principal's realized gross profit is given by $s \in\{\underline{s}, \bar{s}\}$, (iii) for given messages $\widehat{e}$ and $\widehat{s}, \tau_{0}(\widehat{e}, \widehat{s})$ denotes the payment to the agent if the principal's realized gross profit is zero (which is the case when the agent leaves), (iv) due to the agent's limited liability $\tau(\widehat{e}, \widehat{s} ; s), \tau_{0}(\widehat{e}, \widehat{s}) \geq 0$

\footnotetext{
${ }^{24}$ Similar to above, ex-ante participation by both the agent and the principal is always ensured.
} 
holds for all $\widehat{e}, \widehat{s}, s$, and (v) the agent's incentive compatibility condition

$$
\max \left\{\tau(e, s ; s), \lambda e+\tau_{0}(e, s)\right\} \geq \max \left\{\tau(\widehat{e}, \widehat{s} ; s), \lambda e+\tau_{0}(\widehat{e}, \widehat{s})\right\}
$$

is satisfied for all $e, \widehat{e} \in[0,1]$ and for all $s, \widehat{s} \in\{\underline{s}, \bar{s}\} .{ }^{25}$ Denote the subset of contracts where the contractually promised payments do not vary in the agent's messages (both on and off the equilibrium path) by $\widetilde{T}$.

Proposition 6 (Restriction to $T^{+}$is without loss of generality) For any contract in $T$, there exists a contract in $\widetilde{T}$ that leads to the same equilibrium outcome (i.e., effort level, ex-post participation decisions, and payoffs). Moreover, for any contract in $\widetilde{T}$ that either (i) specifies a strictly positive payment when the gross profit is zero or (ii) specifies a payment when the gross profit is $\underline{s}$ that is strictly larger than the payment when the gross profit is $\bar{s}$, there exists a contract in $T^{+}$that yields the principal a strictly higher payoff.

Renegotiation We have so far assumed that the principal can commit to the terms of the initial contract. However, since $\lambda<\underline{s}<\bar{s}$, whenever she offers a partial-separation contract, this leads to an inefficient outcome ex-post, such that the parties have an incentive to renegotiate. ${ }^{26}$ Specifically, after effort provision, the principal might be tempted to propose altered payments that induce participation in all states. ${ }^{27}$ As this would lead to a larger surplus expost, there always exist new payments that would make both parties better off. When the principal cannot credibly commit not to renegotiate, without loss of generality, one can restrict attention to initial contracts that are renegotiation-proof (in the sense of inducing an equilibrium outcome that is ex-post pareto-efficient). Clearly, in our framework this is the case when the initial contract induces the agent to stay in both states or, using our terminology, when it is of the no-separation type. This observation in combination with Proposition 6 implies that,

\footnotetext{
${ }^{25}$ Note that when sending his messages between dates 3 and 4 , the agent knows the state, and effort costs are already sunk, which explains the form of the agent's incentive compatibility condition.

${ }^{26}$ Beginning with Fudenberg and Tirole (1990) and Ma (1991), there is a substantial literature on renegotiation in static moral hazard problems with a risk-averse agent. In these models, participation by the agent is not an issue. Rather, while from an ex-ante incentive perspective the agent should bear some risk, from an expost perspective the agent should be fully insured. More closely related, Netzer and Scheuer (2008) augment Fudenberg and Tirole's (1990) setup by assuming that the agent is free to leave. They study how ex-post competition among principals affects the risk versus incentive trade-off in a setting with no commitment.

${ }^{27}$ In equilibrium, the principal correctly anticipates the agent's outside option, and hence may be tempted to raise the respective payment to match it.
} 
when renegotiation cannot be ruled out, without loss of generality one can restrict attention to no-separation contracts $t \in T_{2}$. In this case, Proposition 2 (which characterizes optimal no-separation contracts) also characterizes the globally optimal contract. As a result, all of the properties of this contract type (such as first-best incentives and surplus, or non-responsiveness) would remain equilibrium features if renegotiation could not be ruled out.

\section{Conclusion}

We modify the standard moral hazard model under risk-neutrality and limited liability by assuming one-sided commitment only: while the principal is able to commit to the contract terms, the agent is free to leave (and hence, he will only stay with the principal if he has an incentive to do so). We consider the case that the agent's ex-post outside option is endogenous (i.e., increasing in effort). This leads to results which are in stark contrast to the standard case with full commitment. In particular, as long as first-best effort is not too large, the principal finds it optimal to provide first-best effort incentives and to structure the contract such that the agent always stays (and hence does not take up his outside option); resulting in the first-best social surplus. If, on the other hand, first-best effort is sufficiently large, the principal optimally proposes a contract that might be non-responsive to changes in the underlying parameters or that might induce (inefficient) separation in some states of the world. Under the latter type of (partial-separation) contract, the agent may be harmed in equilibrium as his outside option becomes (ceteris paribus) more attractive. Finally, we also highlight that our results are driven by the combination of the agent's freedom to leave and the endogeneity of his outside option.

In future work it would be interesting to analyze situations where the effort provided by the agent inside the relationship with the principal actually harms the agent's ex-post outside option. For example, this would be the case if by tailoring his human capital to the needs of the principal, the agent reduces his outside opportunities. Recently, this issue has been investigated by Rasul and Sonderegger (2010), however, in the context of an adverse selection model where an ex-ante investment affects some ex-post outside option. 


\section{Appendix}

Lemmas 1 and 2 and Propositions 3 and 5 follow immediately from the discussion in the text and require no separate formal proof.

\section{A Proof of Lemma 3}

Ad (i): We prove the claim by showing that (a) for $\frac{t}{\lambda} \in[0,1)$ we have $e^{*}(t) \neq \frac{t}{\lambda}$, and (b) for $\frac{\bar{t}}{\lambda} \in[0,1)$ we have $e^{*}(t) \neq \frac{\bar{t}}{\lambda}$. First, suppose $\frac{\bar{t}}{\lambda} \in(0,1)$. In this case, the set $E_{0}(t)=\left(\frac{\bar{t}}{\lambda}, 1\right]$ is non-empty (see Lemma 1), and $e=\frac{\bar{t}}{\lambda}$ cannot be the agent's optimal choice of effort because, on the one hand, $U(e, t)$ is continuous and piece-wise concave and, on the other hand, Lemma 2 (iii) implies $\frac{\partial U_{1}\left(\frac{\bar{t}}{\lambda}, t\right)}{\partial e} \geq 0 \Rightarrow \frac{\partial U_{0}\left(\frac{\bar{t}}{\lambda}, t\right)}{\partial e}>0$ (that is, together these observations imply that there exist effort levels slightly smaller or larger than $\frac{\bar{t}}{\lambda}$ that yield the agent a strictly larger payoff).

Second, suppose $\frac{t}{\lambda} \in(0,1)$. In this case, the set $E_{1}(t)=\left(\frac{t}{\lambda}, \min \left(\frac{\bar{t}}{\lambda}, 1\right)\right]$ is non-empty (see Lemma 1). Moreover, Lemma 2 (iii) implies $\frac{\partial U_{2}\left(\frac{t}{\lambda}, t\right)}{\partial e} \geq 0 \Rightarrow \frac{\partial U_{1}\left(\frac{t}{\lambda}, t\right)}{\partial e}>0$. On the one hand, if both $\frac{t}{\lambda} \in(0,1)$ and $\underline{t} \neq \bar{t}$ hold, similar to above, these considerations in combination with the continuity and piece-wise concavity of $U(e, t)$ imply that $e=\frac{t}{\lambda}$ cannot be the agent's optimal choice of effort. On the other hand, if both $\frac{t}{\lambda} \in(0,1)$ and $\underline{t}=\bar{t}$ hold, we have $E_{1}(t)=\left(\frac{t}{\lambda}, \frac{t}{\lambda}\right]=\varnothing$ and $E_{0}(t)=\left(\frac{t}{\lambda}, 1\right] \neq \varnothing$. Moreover, in this case it follows from (5) that $\frac{\partial U_{2}\left(\frac{t}{\lambda}, t\right)}{\partial e}=-\frac{t}{\lambda}<-\frac{t}{\lambda}+\lambda=\frac{\partial U_{0}\left(\frac{t}{\lambda}, t\right)}{\partial e}$. Hence, again similar to above, the continuity and piecewise concavity of $U(e, t)$ imply that $e=\frac{t}{\lambda}$ cannot be the agent's optimal choice of effort. Finally, suppose $\frac{t}{\lambda}=0\left(\frac{\bar{t}}{\lambda}=0\right)$, in which case it follows that $e^{*}(t) \neq \frac{t}{\lambda}\left(e^{*}(t) \neq \frac{\bar{t}}{\lambda}\right)$ because $\frac{\partial U_{1}(0, t)}{\partial e}, \frac{\partial U_{0}(0, t)}{\partial e}>0$ holds for all $t \in T^{+}$.

Ad (ii): In a first step, suppose that, for a given $t \in T^{+}$, we have $e^{*}(t) \in E_{2}(t)$, where, by definition, $E_{2}(t)=\left[0, \min \left(\frac{t}{\lambda}, 1\right)\right]$. The discussion in the proof of Lemma 3 (i) above implies that in this case it must hold that $\frac{t}{\lambda}>0$. On the one hand, if $\frac{t}{\lambda}<1$, then the concavity of $U_{2}(e, t)$ in combination with $e^{*}(t) \neq \frac{t}{\lambda}$ (see the proof of Lemma 3 (i) above) immediately implies that $e^{*}(t)=e_{2}^{*}(t) \equiv \Delta t<\frac{t}{\lambda}$ has to hold. On the other hand, if $\frac{t}{\lambda} \geq 1$, then the concavity of $U_{2}(e, t)$ in combination with $E_{2}(t)=[0,1]$ implies $e^{*}(t)=\min \left(e_{2}^{*}(t), 1\right)$. Hence, to summarize $e^{*}(t) \in E_{2}(t)$ implies $e^{*}(t)=\min \left(e_{2}^{*}(t), 1\right)$. In a second step, following analogous lines of reasoning, it is straightforward to show that $e^{*}(t) \in E_{1}(t)$ implies $e^{*}(t)=\min \left(e_{1}^{*}(t), 1\right)$ and that $e^{*}(t) \in E_{0}(t)$ implies $e^{*}(t)=\min \left(e_{0}^{*}(t), 1\right)$, which concludes the proof of the Lemma. 


\section{B Proof of Proposition 1}

For given $t \in T^{+}$, we first characterize whether $e^{*}(t) \in E_{2}(t), e^{*}(t) \in E_{1}(t)$, or $e^{*}(t) \in E_{0}(t)$ holds (i.e., we characterize which equilibrium ex-post participation decisions emerge for a given contract), and, subsequently, we employ Lemma 3 to characterize $e^{*}(t)$. We proceed in six steps.

Step 1. By using basic properties of the agent's payoff function (see Lemma 2), one can show that, for some contracts $t$, some ex-post participation decisions cannot emerge in equilibrium. Put differently, in the following we show that in some regions of the contract space $T^{+}$it cannot be optimal for the agent to choose his effort from certain regions of the effort space. To this end, define two functions $f(\bar{t}) \equiv \frac{\lambda}{1+\lambda} \cdot \bar{t}$ and $g(\bar{t}) \equiv \frac{\lambda^{2}}{1+2 \lambda}+\frac{\lambda}{1+2 \lambda} \cdot \bar{t}$, and a threshold value $h \equiv \frac{\lambda^{2}}{1+\lambda}$ (see Figure 10, which depicts the lines $f, g$, and $h$ in the contract space). The following lemma (which is proven at the end of this section) will be useful.

Lemma 4 Suppose $t \in T^{+}$. Then

(i) $e^{*}(t) \notin E_{1}(t)$ if either $\bar{t} \leq h$ or $g(\bar{t}) \leq \underline{t}<\lambda$ holds, and

(ii) $e^{*}(t) \notin E_{2}(t)$ if both $\underline{t} \leq f(\bar{t})$ and $\underline{t}<\lambda$ hold.

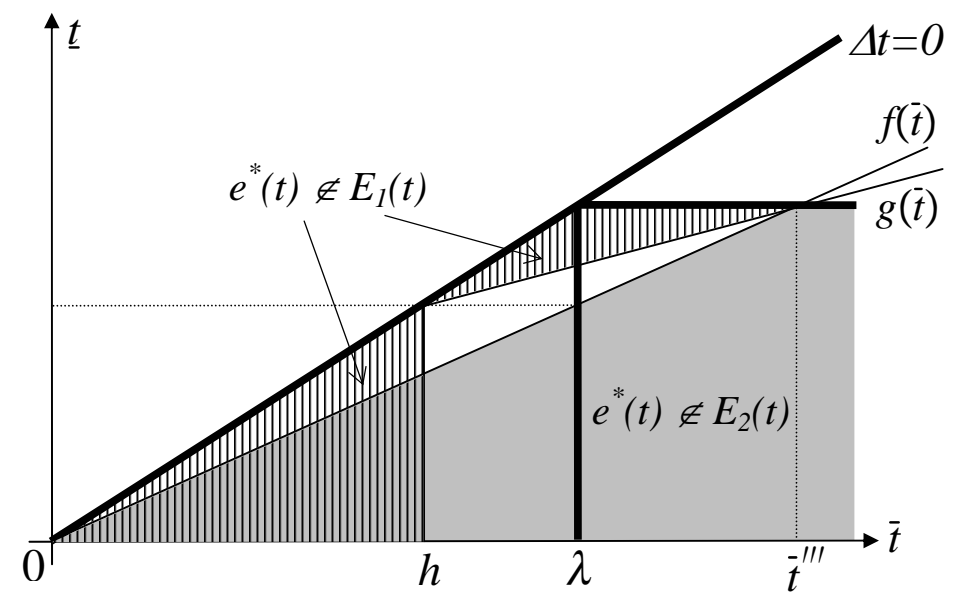

Figure 10: Illustration of Lemma 4

Lemma 4 in combination with Lemma 2 pins down the equilibrium ex-post participation decisions of the agent for large parts of the contract space. This is illustrated by Figure 11 that combines the information contained in Figures 3 and 10. In particular, Figure 11 depicts 


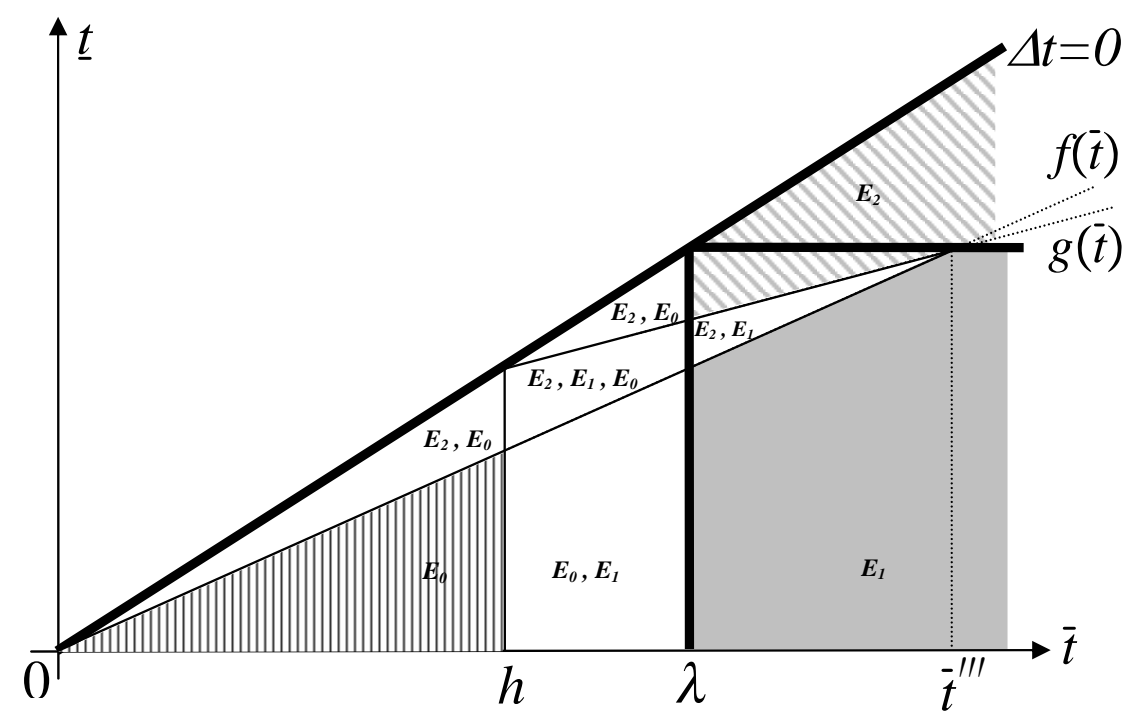

Figure 11: Only in the set $\widehat{T}$ of contracts (the non-shaded, white part of $T^{+}$) Lemma 4 in combination with Lemma 2 does not yet uniquely pin down the equilibrium ex-post participation decisions (for ease of exposition, the dependency of $E_{j}(t)$ on $t$, for $j=\{0,1,2\}$, is suppressed).

the candidate effort intervals in which the agent's optimal effort $e^{*}(t)$ may be located for a given $t$. For example, consider the grey-shaded area of Figure 11. In this region of the contract space, Lemma 2 implies $E_{2}(t)=\left[0, \frac{t}{\lambda}\right], E_{1}(t)=\left(\frac{t}{\lambda}, 1\right]$, and $E_{0}(t)=\varnothing$, and Lemma 4 implies $e^{*}(t) \notin E_{2}(t)$; implying that in the grey-shaded area it must be the case that $e^{*}(t) \in E_{1}(t)$. Only in the non-shaded, white parts of Figure 11, Lemma 4 in combination with Lemma 2 does not yet pin down the equilibrium participation decisions (i.e., the respective set $E_{j}(t)$ in which $e^{*}(t)$ is located). Formally, this subset of contracts is defined as $\widehat{T} \equiv\left\{(\underline{t}, \bar{t}) \in T^{+}\right.$such that either (i) $\bar{t} \in[0, h]$ and $\underline{t}>f(\bar{t})$, or (ii) $\bar{t} \in(h, \lambda)$, or (iii) $\bar{t} \in\left[\lambda, \bar{t}^{\prime \prime \prime}\right)$ and $\left.g(\bar{t})>\underline{t}>f(\bar{t})\right\}$.

Step 2. We now restrict attention to the set of contracts $\widehat{T}$ (see Step 1 above). Given $t \in \widehat{T}$, for each non-empty effort interval $E_{j}(t)$ for which $e^{*}(t) \in E_{j}(t)$ has not been ruled out by Lemma 4 (see Figure 11), we now characterize the effort level that is (locally) optimal in $E_{j}(t)$. This will allow us to pin down the maximum payoff the agent may obtain in each of the relevant intervals $E_{j}(t)$.

Lemma 5 Suppose $t \in \widehat{T}$.

(i) If $\underline{t}>f(\bar{t})$ holds, the effort level that is optimal in the set $E_{2}(t)$ is given by $e_{2}^{*}(t)$. In this case, $e_{2}^{*}(t) \in[0,1)$. 
(ii) If both $\bar{t}>h$ and $\underline{t}<g(\bar{t})$ hold, the effort level that is optimal in the set $E_{1}(t)$ is given by $e_{1}^{*}(t)$. In this case, $e_{1}^{*}(t) \in(0,1)$.

(iii) The effort level that is optimal in the set $E_{0}(t)$ is given by (a) $e=1$ if both $\lambda \geq 1$ and $\bar{t}<\lambda$ hold, and (b) $e=\lambda$ if both $\lambda<1$ and $\bar{t}<\lambda^{2}$ hold. If both $\lambda<1$ and $\lambda^{2} \leq \bar{t}<\lambda$ hold, we have $e^{*}(t) \notin E_{0}(t)$.

Lemma 5 (which is proven at the end of this section) allows to characterize the boundaries between the sets $T_{0}, T_{1}$, and $T_{2}$, where these boundaries will be located in $\widehat{T}$. For further reference, note that (5) in combination with the Envelope-Theorem implies

$$
\frac{\partial U_{2}\left(e_{2}^{*}(t), t\right)}{\partial \bar{t}}=e_{2}^{*}(t), \frac{\partial U_{2}\left(e_{2}^{*}(t), t\right)}{\partial \underline{t}}=1-e_{2}^{*}(t), \frac{\partial U_{1}\left(e_{1}^{*}(t), t\right)}{\partial \bar{t}}=e_{1}^{*}(t), \text { and } \frac{\partial U_{1}\left(e_{1}^{*}(t), t\right)}{\partial \underline{t}}=0
$$

for $e_{2}^{*}(t), e_{1}^{*}(t) \in(0,1)$.

Step 3. Next, we identify contracts $t \in \widehat{T}$ that make the agent indifferent between choosing some effort level from the set $E_{0}(t)$ or choosing some effort level from the set $E_{1}(t)$ (which may, in principle, exist if both $\bar{t} \in(h, \lambda)$ and $\underline{t}<g(\bar{t})$ hold (see Lemmas 2 and 4 and Figure 11)). If such contracts exist, the above observations in combination with Lemma 5 imply that such contracts are implicitly defined by $U_{1}\left(e_{1}^{*}(t), t\right)=U_{0}$, where

$$
\begin{gathered}
U_{1}\left(e_{1}^{*}(t), t\right)=U_{0} \Leftrightarrow e_{1}^{*}(t) \bar{t}+\left(1-e_{1}^{*}(t)\right) \lambda e_{1}^{*}(t)-\frac{1}{2}\left(e_{1}^{*}(t)\right)^{2}=U_{0} \\
\Leftrightarrow \frac{(\bar{t}+\lambda)^{2}}{1+2 \lambda}-\frac{1+2 \lambda}{2} \frac{(\bar{t}+\lambda)^{2}}{1+2 \lambda}=U_{0} \Leftrightarrow \frac{(\bar{t}+\lambda)^{2}}{2(1+2 \lambda)}=U_{0} \\
\Leftrightarrow \bar{t}=\bar{t}^{\prime \prime} \equiv \sqrt{2(1+2 \lambda) U_{0}}-\lambda,
\end{gathered}
$$

and where it is straightforward to show that (a) $\bar{t}^{\prime \prime} \in\left(U_{0}, \lambda\right)$ if $\lambda \geq 1$ and (b) $\bar{t}^{\prime \prime} \in\left(U_{0}, \lambda^{2}\right)$ if $\lambda<1$. To summarize, in the set $\widehat{T}$ there indeed exist contracts where the agent is indifferent between choosing an effort level $\min (\lambda, 1) \in E_{0}(t)$ and choosing an effort level $e_{1}^{*}(t) \in E_{1}(t)$. More precisely, the above observations imply that in the set of contracts $\{t \in \widehat{T}$ such that $\bar{t} \in(h, \lambda)$ and $\underline{t}<g(\bar{t})\}$, the agent prefers $\min (\lambda, 1) \in E_{0}(t)$ over $e_{1}^{*}(t) \in E_{1}(t)$ if $\bar{t}<\bar{t}^{\prime \prime}$ holds, while he prefers $e_{1}^{*}(t) \in E_{1}(t)$ over $\min (\lambda, 1) \in E_{0}(t)$ if $\bar{t} \geq \bar{t}^{\prime \prime}$ holds.

Step 4. We now identify contracts $t \in \widehat{T}$ that make the agent indifferent between choosing some effort level from the set $E_{2}(t)$ or choosing some effort level from the set $E_{1}(t)$ (which may, in principle, exist if both $g(\bar{t})>\underline{t}>f(\bar{t})$ and $\bar{t} \in\left(h, \bar{t}^{\prime \prime \prime}\right.$ ) hold (see Lemmas 2 and 4 
and Figure 11). If such contracts exist, then, in analogy to above, Lemma 5 (i) and (ii) imply that they are located on the graph of a function $y(\bar{t}): \bar{t} \rightarrow \underline{t}$ that is implicitly defined by $U_{2}\left(e_{2}^{*}(t), t\right)=U_{1}\left(e_{1}^{*}(t), t\right)$. Moreover, for all contracts $t$ on the graph of $y(\bar{t})$, it holds that (a) $e_{1}^{*}(t)=\frac{\bar{t}+\lambda}{2 \lambda+1} \in(0,1)$ (see Lemma $5(\mathrm{ii})$ ), and (b) $0 \leq e_{2}^{*}(t)=\Delta t<e_{1}^{*}(t)$ which follows from $e_{2}^{*}(t) \in E_{2}(t)=\left[0, \frac{t}{\lambda}\right], e_{1}^{*}(t) \in E_{1}(t)=\left(\frac{t}{\lambda}, \min \left(\frac{\bar{t}}{\lambda}, 1\right)\right]$, and $U_{2}^{\prime}\left(\frac{t}{\lambda}, t\right)<U_{1}^{\prime}\left(\frac{t}{\lambda}, t\right)$ (see Lemma 2 (iii)). Note that $U_{2}\left(e_{2}^{*}(t), t\right)=U_{1}\left(e_{1}^{*}(t), t\right)$, the Implicit-Function Theorem, (14), and the above observations with respect to $e_{2}^{*}(t)$ and $e_{1}^{*}(t)$ imply

$$
\begin{gathered}
\frac{\partial y(\bar{t})}{\partial \bar{t}}=\frac{e_{1}^{*}(t)-e_{2}^{*}(t)}{1-e_{2}^{*}(t)} \in(0,1), \text { and } \\
\frac{\partial^{2} y(\bar{t})}{\partial \bar{t}^{2}}<0 \Leftrightarrow\left(1-e_{2}^{*}(t)\right) \cdot\left(\frac{1}{1+2 \lambda}-1\right)+\left(e_{1}^{*}(t)-e_{2}^{*}(t)\right)<0 \Leftrightarrow \frac{\underline{t}-\lambda}{\left(1-e_{2}^{*}(t)\right)^{2}(1+2 \lambda)}<0,
\end{gathered}
$$

which is satisfied, because for all $t \in \widehat{T}$ we have $\underline{t}<\lambda$ (see Figure 11). Moreover, note that $U_{2}\left(e_{2}^{*}(t), t\right)=U_{1}\left(e_{1}^{*}(t), t\right)$ is satisfied at $t=\left(\bar{t}=\bar{t}^{\prime \prime \prime} \equiv 1+\lambda, \underline{t}=\lambda\right)$. That is, $\lim _{\bar{t} \rightarrow \bar{t}^{\prime \prime \prime}} y(\bar{t})=\lambda$.

Also, for all $\bar{t} \in\left(h, \bar{t}^{\prime \prime \prime}\right)$ we have $y(\bar{t}) \in(f(\bar{t}), g(\bar{t}))$, which follows from the following Intermediate Value Theorem argument. Take some arbitrary $\bar{t} \in\left(h, \bar{t}^{\prime \prime \prime}\right)$ as given. First, suppose that, at the same time, $\underline{t} \leq f(\bar{t})$ holds. In this case, the proof of Lemma 4 (ii) implies that, given such a contract, the agent obtains a strictly higher payoff from choosing some effort level in $E_{1}(t)$ than from choosing any effort level in $E_{2}(t)$. Second, suppose that $\underline{t} \geq g(\bar{t})$ holds. In this case, the proof of Lemma 4 (i) implies that, given such a contract, the agent obtains a strictly higher payoff from choosing some effort level in $E_{2}(t)$ than from choosing any effort level in $E_{1}(t)$. These observations, the Intermediate Value Theorem, and the fact that $U_{1}\left(e_{1}^{*}(t), t\right)$ is independent of $\underline{t}$ while $U_{2}\left(e_{2}^{*}(t), t\right)$ is increasing in $\underline{t}$ imply the claim.

To summarize, the above discussion shows that in the set $\widehat{T}$ there indeed exist contracts where the agent is indifferent between choosing an effort level $e_{2}^{*}(t) \in E_{2}(t)$ and choosing an effort level $e_{1}^{*}(t) \in E_{1}(t)$. More precisely, the above observations imply that in the set of contracts $\left\{t \in \widehat{T}\right.$ such that $g(\bar{t})>\underline{t}>f(\bar{t})$ and $\left.\bar{t} \in\left(h, \bar{t}^{\prime \prime \prime}=1+\lambda\right)\right\}$, the agent prefers $e_{2}^{*}(t) \in E_{2}(t)$ over $e_{1}^{*}(t) \in E_{1}(t)$ if $\underline{t} \geq y(\bar{t})$ holds, while he prefers $e_{1}^{*}(t) \in E_{1}(t)$ over $e_{2}^{*}(t) \in E_{2}(t)$ if $\underline{t}<y(\bar{t})$ holds.

Step 5. Now, we identify contracts $t \in \widehat{T}$ that make the agent indifferent between choosing some effort level from the set $E_{2}(t)$ or choosing some effort level from the set $E_{0}(t)$ (which may, in principle, exist if both $\underline{t}>f(\bar{t})$ and $\bar{t}<\lambda$ hold (see Lemmas 2 (i) and 4 and Figure 11)). If such contracts exist, then, in analogy to above, Lemma 5 (i) and (iii) imply that they are located 
on the graph of a function $x(\bar{t}): \bar{t} \rightarrow \underline{t}$ that is implicitly defined by $U_{2}\left(e_{2}^{*}(t), t\right)=U_{0}$. Moreover, for all contracts $t$ on the graph of $x(\bar{t})$, it holds that (a) $e_{2}^{*}(t)=\Delta t<1$ (see Lemma 5 (i)), and (b) $e_{2}^{*}(t)=0$ if and only if $\Delta t=0$. Hence, the fact that $U_{2}\left(e_{2}^{*}(t), t\right)=U_{0}$ in combination with the Implicit Function Theorem, (14), and the above observations with respect to $e_{2}^{*}(t)$ imply

$$
\begin{gathered}
\frac{\partial x(\bar{t})}{\partial \bar{t}}=-\frac{e_{2}^{*}(t)}{1-e_{2}^{*}(t)}<0(=0) \text { for } e_{2}^{*}(t)>0\left(e_{2}^{*}(t)=0\right), \text { and } \\
\frac{\partial^{2} x(\bar{t})}{\partial \bar{t}^{2}}=-\frac{1}{\left[1-e_{2}^{*}(t)\right]^{2}}<0 .
\end{gathered}
$$

Moreover, note that $U_{2}\left(e_{2}^{*}(t), t\right)=U_{0}$ is satisfied at $t=\left(\bar{t}=\bar{t}^{\prime} \equiv U_{0}, \underline{t}=U_{0}\right)$. That is, $x\left(U_{0}\right)=$ $U_{0}$, and recall from Step 3 that $U_{0}<\bar{t}^{\prime \prime}$ holds. Also, recall from Step 4 that $y(\bar{t}) \in(f(\bar{t}), g(\bar{t}))$ for all $\bar{t} \in\left(h, \bar{t}^{\prime \prime \prime}\right)$, which implies $y\left(\bar{t}^{\prime \prime}\right) \in\left(f\left(\bar{t}^{\prime \prime}\right), g\left(\bar{t}^{\prime \prime}\right)\right)$ (for the definition of $\bar{t}^{\prime \prime}$ see (15)). Hence, for the contract $\widetilde{t} \equiv\left(\underline{t}=y\left(\bar{t}^{\prime \prime}\right), \bar{t}=\bar{t}^{\prime \prime}\right)$ it holds that $U_{2}\left(e_{2}^{*}(\widetilde{t}), \widetilde{t}\right)=U_{1}\left(e_{1}^{*}(\widetilde{t}), \widetilde{t}\right)=U_{0}$ (because contract $\widetilde{t}$ is located on the graph of $y(\bar{t})$ and $\bar{t}=\bar{t}^{\prime \prime}$ holds), which implies $x\left(\bar{t}^{\prime \prime}\right)=y\left(\bar{t}^{\prime \prime}\right)$.

To summarize, in the set $\widehat{T}$ there indeed exist contracts where the agent is indifferent between choosing an effort level $e_{2}^{*}(t) \in E_{2}(t)$ and an effort level $\min (\lambda, 1) \in E_{0}(t)$. More precisely, the above observations imply that in the set of contracts $\{t \in \widehat{T}$ such that $\underline{t}>f(\bar{t})$ and $\left.\bar{t}<\bar{t}^{\prime \prime}\right\}$, the agent prefers $\min (\lambda, 1) \in E_{0}(t)$ over $e_{2}^{*}(t) \in E_{2}(t)$ if either (i) $\bar{t}<U_{0}$ or (ii) $\bar{t} \in\left[U_{0}, \bar{t}^{\prime \prime}\right]$ and $\underline{t}<x(\bar{t})$ holds, while the agent prefers $e_{2}^{*}(t) \in E_{2}(t)$ over $\min (\lambda, 1) \in E_{0}(t)$ otherwise.

Step 6. Taken together, Steps 1 through 5 imply the definitions of the sets $T_{0}, T_{1}$, and $T_{2}$ in Proposition 1 because for $\bar{t} \in\left[\bar{t}^{\prime}, \bar{t}^{\prime \prime}\right]$ we have $b(\bar{t})=x(\bar{t})$, while for $\bar{t} \in\left[\bar{t}^{\prime \prime}, \bar{t}^{\prime \prime \prime}\right)$ we have $b(\bar{t})=y(\bar{t})$ (see Definition 1). That is, for $j=0,1,2$, we have shown that $t \in T_{j} \Rightarrow e^{*}(t) \in E_{j}(t)$, which in combination with Lemma 3(ii) implies $e^{*}(t)=\min \left(e_{j}^{*}(t), 1\right)$. Finally, the properties of $b(\bar{t})$ as stated in Proposition 1 immediately follow from the discussion of the properties of $x(\bar{t})$ and $y(\bar{t})$ above, which concludes the proof.

\section{B.1 Proof of Lemma 4}

Note that $h=\frac{\lambda^{2}}{1+\lambda}<\lambda, g(h)=h, g\left(\bar{t}^{\prime \prime \prime}\right)=\lambda, f(0)=0$ and $f\left(\bar{t}^{\prime \prime \prime}\right)=\lambda$, which implies that the lines $h, g(\bar{t})$, and $f(\bar{t})$ are located exactly as depicted in Figure 10.

$A d$ (i): First, suppose $\bar{t} \leq h$, which is equivalent to $\frac{\partial U_{1}\left(\frac{\bar{t}}{\lambda}, t\right)}{\partial e} \geq 0$ (see (5)). This observation in combination with the concavity of $U_{1}(e, t)$ implies that $U_{1}(e, t)$ is increasing in $e$ for all $e \in E_{1}(t)$. Moreover, recall that $h<\lambda$, and hence $\frac{\bar{t}}{\lambda}<1$; implying $E_{0}(t)=\left(\frac{\bar{t}}{\lambda}, 1\right] \neq \varnothing$ (see 
Lemma 1). These observations in combination with Lemma 2 imply that there exists some effort level in $E_{0}(t)$ that yields the agent a strictly higher payoff than any effort level in $E_{1}(t)$, and hence $e^{*}(t) \notin E_{1}(t)$. Note that in the case $\bar{t}=0$ we have $E_{1}(t)=\varnothing$. Second, suppose $g(\bar{t}) \leq \underline{t}<\lambda$, in which case $E_{1}(t)=\left(\frac{t}{\lambda}, \min \left(\frac{\bar{t}}{\lambda}, 1\right)\right] \neq \varnothing$ (which follows from $\left.\frac{t}{\lambda}<1\right)$ and $\frac{\partial U_{1}\left(\frac{t}{\lambda}, t\right)}{\partial e} \leq 0$ (which is equivalent to $g(\bar{t}) \leq \underline{t}$ ). These observations in combination with the concavity of $U_{1}(e, t)$ imply that $U_{1}(e, t)$ is decreasing in $e$ for all $e \in E_{1}(t)$. Consequently, it follows from Lemma 2 and the fact that $E_{2}(t) \neq \varnothing \forall t \in T^{+}$that there exists some effort level in $E_{2}(t)$ that yields the agent a strictly higher payoff than any effort level in $E_{1}(t)$, and hence $e^{*}(t) \notin E_{1}(t)$.

Ad (ii): Note that $E_{2}(t)=\left[0, \frac{t}{\lambda}\right]$ (which follows from $\left.\underline{t}<\lambda\right)$ and $E_{1}(t)=\left(\frac{t}{\lambda}, \min \left(\frac{\bar{t}}{\lambda}, 1\right)\right]$, where $E_{1}(t) \neq \varnothing$ unless $\underline{t}=\bar{t}=0$ (see Lemmas 1 and 2). However, if $\underline{t}=\bar{t}=0$, it follows from $\frac{\partial U_{1}(0, t)}{\partial e}, \frac{\partial U_{0}(0, t)}{\partial e}>0 \forall t \in T^{+}$that $e^{*}(t) \notin E_{2}(t)$. Hence, in the following suppose $\underline{t}>0$. Note that $\frac{\partial U_{2}\left(\frac{t}{\lambda}, t\right)}{\partial e} \geq 0$ (which is equivalent to $\underline{t} \leq f(\bar{t})$ ), which in combination with the concavity of $U_{2}(e, t)$ implies that $U_{2}(e, t)$ is increasing in $e$ for all $e \in E_{2}(t)$. Consequently, it follows from Lemma 2(iii) that there exists some effort level in $E_{1}(t)$ that yields the agent a strictly higher payoff than any effort level in $E_{2}(t)$, and hence $e^{*}(t) \notin E_{2}(t)$.

\section{B.2 Proof of Lemma 5}

$A d$ (i): From (5) we have $\frac{\partial U_{2}\left(\frac{t}{\lambda}, t\right)}{\partial e}<0$ for all $\underline{t}>f(\bar{t})$, where, for all $t \in \widehat{T}$, we have $\frac{t}{\lambda}<1$. These observations in combination with the concavity of $U_{2}(e, t)$ and $\frac{\partial U_{2}(0, t)}{\partial e} \geq 0$ imply that the effort level that is optimal in $E_{2}(t)=\left[0, \frac{t}{\lambda}\right]$ is defined by the respective first-order condition, and hence is given by $e_{2}^{*}(t)=\Delta t$, where $e_{2}^{*}(t) \in\left[0, \frac{t}{\lambda}\right)$ holds.

Ad (ii): Using Lemma 2 it can be shown that $\frac{\partial U_{1}\left(\frac{t}{\lambda}, t\right)}{\partial e}>0>\frac{\partial U_{1}\left(\frac{\bar{t}}{\lambda}, t\right)}{\partial e}$ for all $t \in \widehat{T}$ satisfying $\bar{t}>h$ and $\underline{t}<g(\bar{t})$, where we have $\frac{t}{\bar{\lambda}}<1$. These observations in combination with the concavity of $U_{1}(e, t)$ imply that the effort level that is optimal in $E_{1}(t)=\left(\frac{t}{\lambda}, \min \left(\frac{\bar{t}}{\lambda}, 1\right)\right]$ is defined by the respective first-order condition, and hence is given by $e_{1}^{*}(t)=\frac{\bar{t}+\lambda}{2 \lambda+1}$ if $\frac{\bar{t}+\lambda}{2 \lambda+1} \leq 1$ holds. This is indeed the case because for all $t \in \widehat{T}$ we have $\bar{t}<1+\lambda$, and hence $\frac{\bar{t}+\lambda}{2 \lambda+1}<1$.

Ad (iii): First, suppose that both $\lambda \geq 1$ and $\bar{t}<\lambda$ hold. In this case, we have $\frac{\bar{t}}{\lambda}<1$ and $\frac{\partial U_{0}(1, t)}{\partial e}=\lambda-1 \geq 0$ (see $(5)$ ). These observations in combination with the concavity of $U_{0}(e, t)$ imply that the effort level that is optimal in $E_{0}(t)=\left(\frac{\bar{t}}{\lambda}, 1\right]$ is given by 1 , which proves part (a). Second, suppose that $\lambda<1$ holds. Recall that $\bar{t}<\lambda$ implies $\frac{\bar{t}}{\lambda}<1$, and 
hence $E_{0}(t)=\left(\frac{\bar{t}}{\lambda}, 1\right] \neq \varnothing$. Note that $\frac{\partial U_{0}\left(\frac{\bar{t}}{\lambda}, t\right)}{\partial e}>\frac{\partial U_{1}\left(\frac{\bar{t}}{\lambda}, t\right)}{\partial e}$ for $\bar{t}>0$ and $\frac{\partial U_{0}\left(\frac{\bar{t}}{\lambda}, t\right)}{\partial e}=\frac{\partial U_{1}\left(\frac{\bar{t}}{\lambda}, t\right)}{\partial e}$ for $\bar{t}=0$ (see Lemma 2). Moreover, from (5), we have $0 \geq \frac{\partial U_{0}\left(\frac{\bar{t}}{\lambda}, t\right)}{\partial e}$ if $\bar{t} \geq \lambda^{2}$. Hence, for $\bar{t} \geq \lambda^{2}$ we have $e^{*}(t) \notin E_{0}(t)$, which proves part (c). With respect to part (b), note that we have $h<\lambda^{2}<\lambda<1$. Moreover, from (5) we have $\frac{\partial U_{0}\left(\frac{\bar{t}}{\lambda}, t\right)}{\partial e}>0>\frac{\partial U_{0}(1, t)}{\partial e}$ for $\bar{t}<\lambda^{2}$. Hence, for $\bar{t}<\lambda^{2}$, these observations in combination with the concavity of $U_{0}(e, t)$ imply that the effort level that is optimal in $E_{0}(t)=\left(\frac{\bar{t}}{\lambda}, 1\right]$ is defined by the respective first-order condition, and hence it is given by $\lambda$, which proves part (b).

\section{Proof of Proposition 2}

The discussion above the Proposition immediately implies that the principal's problem of maximizing (8) can be transformed in a one-dimensional problem of the following form:

$$
\max _{\Delta t \in[0,1]}\{\Delta t \cdot(\Delta s-\Delta t)+\underline{s}-\underline{t}(\Delta t)\},
$$

where, for a given $\Delta t$, the payment in the bad state $\underline{t}(\Delta t)$ has to be such that the contract $(\underline{t}=\underline{t}(\Delta t), \bar{t}=\Delta t+\underline{t}(\Delta t))$ is on the graph of $b(\bar{t})$.

Ad (i): Part (i) immediately follows from (8) in combination with the discussion above the Proposition.

Ad (ii): In a first step towards solving (20), we now derive the functional form of $\underline{t}(\Delta t)$. First, it follows from Definition 1 that, for $\bar{t} \in\left[\bar{t}^{\prime}, \bar{t}^{\prime \prime}\right]$, on the graph of $b(\bar{t})$ it holds that $\frac{1}{2}(\Delta t)^{2}+\underline{t}=\bar{t}^{\prime}$. Hence, $\underline{t}(\Delta t)=\bar{t}^{\prime}-\frac{1}{2}(\Delta t)^{2}$ for $\Delta t \in\left[0, \Delta t^{\prime \prime}\right]$, where $\Delta t^{\prime \prime} \equiv \bar{t}^{\prime \prime}-b\left(\bar{t}^{\prime \prime}\right)<1$. Second, it follows from Definition 1 that, for $t \in\left[\bar{t}^{\prime \prime}, \bar{t}^{\prime \prime \prime}\right]$, on the graph of $b(\bar{t})$ it holds that

$$
\frac{1}{2}(\Delta t)^{2}+\underline{t}=\frac{(\bar{t}+\lambda)^{2}}{2(1+2 \lambda)} \Leftrightarrow 0=\underline{t}^{2}-\underline{t} \cdot[2 \lambda+2-2 \Delta t]+\left[2 \lambda \Delta t+\lambda^{2}-2 \lambda(\Delta t)^{2}\right],
$$

where the equivalence follows from the identity $\bar{t}=\Delta t+\underline{t}$ and rearranging terms. Note that (21) is a quadratic equation in $\underline{t}$. This quadratic equation has two solutions, only one of which is, however, admissible because for all contracts on the graph of $b(\bar{t})$ it holds that $\underline{t} \leq \lambda$.

Hence,

$$
\underline{t}(\Delta t)= \begin{cases}\bar{t}^{\prime}-\frac{1}{2}(\Delta t)^{2} & \text { if } \Delta t \in\left[0, \Delta t^{\prime \prime}\right], \text { and } \\ {[\lambda-(\sqrt{1+2 \lambda}-1)]+\Delta t \cdot[\sqrt{1+2 \lambda}-1]} & \text { if } \Delta t \in\left[\Delta t^{\prime \prime}, 1\right] .\end{cases}
$$

To solve (20), we now proceed in three steps. First, we derive the optimal $\Delta t$ in the interval $\left[0, \Delta t^{\prime \prime}\right]$. Second, we derive the optimal $\Delta t$ in the interval $\left[\Delta t^{\prime \prime}, 1\right]$. Note that $(20)$ in 
combination with $(22)$ implies that both in the interval $\left[0, \Delta t^{\prime \prime}\right]$ and the interval $\left[\Delta t^{\prime \prime}, 1\right]$ the principal's objective function is strictly concave in $\Delta t$. Third, building on these preliminary results we characterize the optimal $\Delta t$ as a function of $\Delta s$.

Step 1. (20) and (22) imply that in the interval $\left[0, \Delta t^{\prime \prime}\right]$ the first-order condition of (20) with respect to $\Delta t$ is satisfied at $\Delta t=\Delta s$. Hence, in the interval $\left[0, \Delta t^{\prime \prime}\right]$ the optimal $\Delta t$ is given by

$$
\Delta t= \begin{cases}\Delta s & \text { if } \Delta s<\Delta t^{\prime \prime}, \text { and } \\ \Delta t^{\prime \prime} & \text { if } \Delta s \geq \Delta t^{\prime \prime} .\end{cases}
$$

Recall that $\Delta t^{\prime \prime}<1$.

Step 2. (20) and (22) imply that in the interval $\left[\Delta t^{\prime \prime}, 1\right]$ the first-order condition of $(20)$ with respect to $\Delta t$ is satisfied at $\Delta t=\frac{\Delta s-\sqrt{1+2 \lambda}+1}{2}$. Hence, in the interval $\left[\Delta t^{\prime \prime}, 1\right]$ the optimal $\Delta t$ is given by

$$
\Delta t= \begin{cases}\Delta t^{\prime \prime} & \text { if } \Delta s \leq \Delta s_{I}, \text { and } \\ \min \left\{\frac{\Delta s-\sqrt{1+2 \lambda}+1}{2}, 1\right\} & \text { if } \Delta s>\Delta s_{I},\end{cases}
$$

where $\Delta s_{I} \equiv 2 \Delta t^{\prime \prime}+\sqrt{1+2 \lambda}-1$ and where $\Delta s>\Delta s_{I} \Leftrightarrow \frac{\Delta s-\sqrt{1+2 \lambda}+1}{2}>\Delta t^{\prime \prime}$. Importantly, note that $\Delta t^{\prime \prime}<\Delta s_{I}=2 \Delta t^{\prime \prime}+\sqrt{1+2 \lambda}-1$.

Step 3. We can now use the preliminary results obtained in Steps 1 and 2 to characterize the effort level that the principal wants to induce for a given $\Delta s .^{28}$ First, consider $\Delta s \in\left(0, \Delta t^{\prime \prime}\right)$. For such $\Delta s, \Delta t=\Delta s<\Delta t^{\prime \prime}$ is optimal in the interval $\left[0, \Delta t^{\prime \prime}\right]$ (see (23)), while $\Delta t=\Delta t^{\prime \prime}$ is optimal in the interval $\left[\Delta t^{\prime \prime}, 1\right]$ (see $\left.(24)\right)$. As $\Delta t=\Delta t^{\prime \prime}$ would also have been feasible in the interval $\left[0, \Delta t^{\prime \prime}\right], \Delta t=\Delta s$ must be globally optimal (i.e., in the interval $\Delta s \in\left(0, \Delta t^{\prime \prime}\right)$ the principal optimally induces $\Delta t=\Delta s)$. Second, consider $\Delta s \in\left[\Delta t^{\prime \prime}, \Delta s_{I}\right]$. For such $\Delta s$, both in the interval $\left[0, \Delta t^{\prime \prime}\right]$ and the interval $\left[\Delta t^{\prime \prime}, 1\right]$ the optimal $\Delta t$ is given by $\Delta t^{\prime \prime}$ (see (23) and (24)), and hence, for all $\Delta s \in\left(0, \Delta s_{I}\right]$, the optimal $\Delta t$ is given by $\Delta t^{\prime \prime}$. To summarize, for $\Delta s \in$ $\left(0, \Delta s_{I}\right]$, the principal induces $\Delta t=\min \left\{\Delta s, \Delta t^{\prime \prime}\right\}$. Finally, consider $\Delta s \in\left(\Delta s_{I}, \infty\right)$. For such $\Delta s, \Delta t=\Delta t^{\prime \prime}$ is optimal in the interval $\left[0, \Delta t^{\prime \prime}\right]$ (see (23)), while $\Delta t=\min \left\{\frac{\Delta s-\sqrt{1+2 \lambda}+1}{2}, 1\right\}>$ $\Delta t^{\prime \prime}$ is optimal in the interval $\left[\Delta t^{\prime \prime}, 1\right]$ (see (24)). As $\Delta t=\Delta t^{\prime \prime}$ would also have been feasible in the interval $\left[\Delta t^{\prime \prime}, 1\right], \Delta t=\min \left\{\frac{\Delta s-\sqrt{1+2 \lambda}+1}{2}, 1\right\}$ must be globally optimal (i.e., in the interval $\Delta s \in\left(\Delta s_{I}, \infty\right)$ the principal optimally induces $\left.\Delta t=\min \left\{\frac{\Delta s-\sqrt{1+2 \lambda}+1}{2}, 1\right\}\right)$, which concludes

\footnotetext{
${ }^{28}$ Intuitively, we now show the following: whenever the optimal $\Delta t$ in the interval $\left[0, \Delta t^{\prime \prime}\right]$ (in the interval $\left.\left[\Delta t^{\prime \prime}, 1\right]\right)$ is in the interior of this interval, the optimal $\Delta t$ in the interval $\left[\Delta t^{\prime \prime}, 1\right]$ (in the interval $\left[0, \Delta t^{\prime \prime}\right]$ ) is equal to $\Delta t^{\prime \prime}$. As $\Delta t^{\prime \prime}$ is an element of both intervals, this implies that whenever the $\Delta t$ that is optimal in one of the two subsets is interior it is also globally optimal in the entire interval $[0,1]$.
} 
the proof.

\section{Proof of Proposition 4}

In the following, we characterize the globally optimal contract across the $(\underline{s}, \bar{s})$-space. At the end of the proof, we show that contracts $t \in T_{0}$ can never be optimal. Consequently, for given $(\underline{s}, \bar{s})$, we now determine whether the globally optimal contract is given (a) by the optimal no-separation contract as characterized in Proposition 2 (which we denote by $t_{2}^{*}(\underline{s}, \bar{s})$ ) or (b) by the optimal partial-separation contract as characterized in Proposition 3 (which we denote by $\left.t_{1}^{*}(\underline{s}, \bar{s})\right)$. Define (see $\left.(8)\right)$ :

$$
D(\underline{s}, \bar{s})=\Pi\left(t_{1}^{*}(\underline{s}, \bar{s})\right)-\Pi\left(t_{2}^{*}(\underline{s}, \bar{s})\right),
$$

and hence contract $t_{1}^{*}(\underline{s}, \bar{s})$ is globally optimal when $D(\underline{s}, \bar{s}) \geq 0$ holds, while contract $t_{2}^{*}(\underline{s}, \bar{s})$ is globally optimal when $D(\underline{s}, \bar{s}) \leq 0$ holds. Note that $D(\underline{s}, \bar{s})$ is continuous in $\underline{s}$ and $\bar{s}$. In the following, we characterize $D(\underline{s}, \bar{s})$ across the $(\underline{s}, \bar{s})$-space. To do so, we proceed in six steps (for an illustration, see Figure 12).

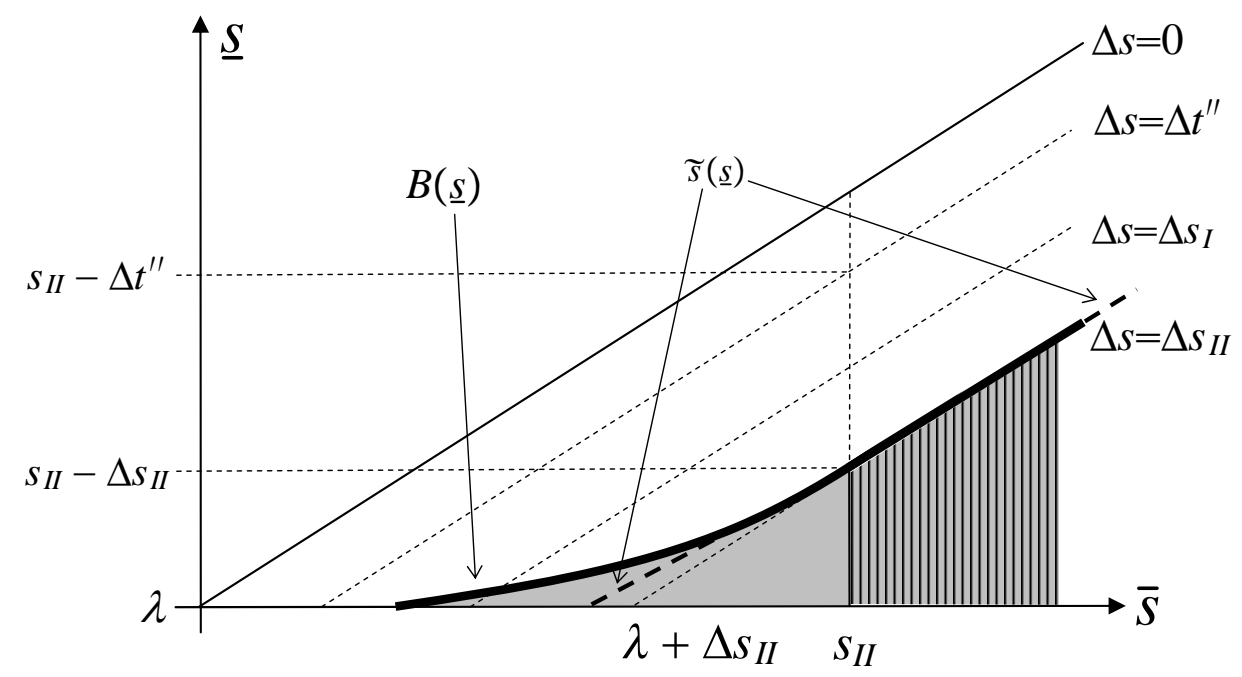

Figure 12: Illustration of the proof of Proposition 4

Step 1. Suppose that both $\bar{s}<s_{I I}$ and $\Delta s \geq \Delta s_{I I}$ hold. This parameter region is nonempty because it can be shown that $\Delta s_{I I}+\lambda<s_{I I}$ holds (see Figure 12). The discussion above Proposition 4 implies: 
Lemma $6 D(\underline{s}, \bar{s})>0$ if both $\bar{s}<s_{I I}$ and $\Delta s \geq \Delta s_{I I}$ hold (where pairs $(\underline{s}, \bar{s})$ satisfying these conditions indeed exist).

Step 2. Now, suppose that $\bar{s} \geq s_{I I}$ holds. In this region, Proposition 3 implies $t_{1}^{*}(\underline{s}, \bar{s})=$ $\left(\underline{t}=0, \bar{t}=\bar{t}^{\prime \prime \prime}\right)$, which would lead the agent to choose an effort level of 1 ; implying that $\bar{s}$ would obtain with certainty. Two cases with respect to $t_{2}^{*}(\underline{s}, \bar{s})$ may arise. First, if $\Delta s \geq \Delta s_{I I}$, then under the optimal no-separation contract exactly the same outcome as under $t_{1}^{*}(\underline{s}, \bar{s})$ would obtain (see Proposition 2(ii)): the agent would exert an effort level of $1, \bar{s}$ would obtain with certainty, and the principal's payoff would be identical to the payoff under the optimal partial-separation contract because under both contracts the payment by the principal in the good state would be given by $\bar{t}^{\prime \prime \prime}$. Hence, in the parameter region where both $\bar{s} \geq s_{I I}$ and $\Delta s \geq \Delta s_{I I}$ hold, the principal is indifferent between $t_{1}^{*}(\underline{s}, \bar{s})$ and $t_{2}^{*}(\underline{s}, \bar{s})$. Second, if, however, $\Delta s<\Delta s_{I I}$ holds, then the contract $t_{2}^{*}(\underline{s}, \bar{s})$ is strictly optimal, which follows from the following "revealed preference"-argument. In this parameter region, the principal could have chosen the contract $\left(\underline{t}=b\left(\bar{t}^{\prime \prime \prime}\right), \bar{t}=\bar{t}^{\prime \prime \prime}\right) \in T_{2}$, which would have yielded her the same payoff as the contract $t_{1}^{*}(\underline{s}, \bar{s})=\left(\underline{t}=0, \bar{t}=\bar{t}^{\prime \prime \prime}\right)$. The fact that the principal chooses some different contract $t_{2}^{*}(\underline{s}, \bar{s})$ (see Proposition 2) implies that, by doing so, she obtains a strictly higher payoff. To summarize (for an illustration, see again Figure 12):

Lemma $7 D(\underline{s}, \bar{s})=0$ if both $\bar{s} \geq s_{I I}$ and $\Delta s \geq \Delta s_{I I}$ hold, and $D(\underline{s}, \bar{s})<0$ if both $\bar{s} \geq s_{I I}$ and $\Delta s<\Delta s_{I I}$ hold.

Step 3. Now, suppose that both $\bar{s} \leq s_{I I}$ and $\Delta s \leq \Delta t^{\prime \prime}$ hold. In this region, it will turn out that $D(\underline{s}, \bar{s})<0$ holds. To see this, note that, for $\Delta s \leq \Delta t^{\prime \prime}$, the contract $t_{2}^{*}(\underline{s}, \bar{s})$ implies first-best effort and the equilibrium payoff of the agent is equal to $\bar{t}^{\prime}$ (see Proposition 2 and Definition 1$)$. We now show that, given the contract $t_{1}^{*}(\underline{s}, \bar{s})$, the principal's payoff would be strictly smaller, which implies the claim. First, note that if $\bar{s} \in\left(s_{I}, s_{I I}\right]$, then, given $t_{1}^{*}(\underline{s}, \bar{s})$, the agent's equilibrium payoff is given by $\frac{(\bar{s}+\lambda)^{2}}{8(1+2 \lambda)}$, which is strictly increasing in $\bar{s}$, and $\frac{\left(s_{I}+\lambda\right)^{2}}{8(1+2 \lambda)}=\bar{t}^{\prime}$. Hence, for all $\bar{s} \in\left(s_{I}, s_{I I}\right]$, compared to $t_{2}^{*}(\underline{s}, \bar{s})$, the contract $t_{1}^{*}(\underline{s}, \bar{s})$ implies a higher equilibrium payoff for the agent (but cannot imply a higher social surplus as $t_{2}^{*}(\underline{s}, \bar{s})$ induces first-best effort). Hence, $t_{2}^{*}(\underline{s}, \bar{s})$ is strictly optimal from the principal's perspective. Second, if $\bar{s} \leq s_{I}$ holds, the contract $t_{2}^{*}(\underline{s}, \bar{s})$ is strictly optimal for the following reasons. While $t_{2}^{*}(\underline{s}, \bar{s})$ induces first-best effort $e_{2}^{*}\left(t_{2}^{*}(\underline{s}, \bar{s})\right)$, contract $t_{1}^{*}(\underline{s}, \bar{s})$ induces a strictly larger 
effort level $e_{1}^{*}\left(t_{1}^{*}(\underline{s}, \bar{s})\right)$ because $e_{2}^{*}\left(t_{2}^{*}(\underline{s}, \bar{s})\right) \leq e_{2}^{*}\left(\underline{t}^{\prime \prime}, \bar{t}^{\prime \prime}\right)<e_{1}^{*}\left(\underline{t}^{\prime \prime}, \bar{t}^{\prime \prime}\right)=e_{1}^{*}\left(t_{1}^{*}(\underline{s}, \bar{s})\right)$ holds: the first inequality follows from Proposition 2 because we currently consider a range of parameters where $\Delta s \leq \Delta t^{\prime \prime}=\bar{t}^{\prime \prime}-\underline{t}^{\prime \prime}$ holds. The second inequality holds because (i) $\bar{t}^{\prime \prime}<\lambda$ implies $E_{2}\left(\underline{t}^{\prime \prime}, \bar{t}^{\prime \prime}\right)=\left[0, \frac{\underline{t}^{\prime \prime}}{\lambda}\right]$ and $E_{1}\left(\underline{t}^{\prime \prime}, \bar{t}^{\prime \prime}\right)=\left(\frac{t^{\prime \prime}}{\lambda}, \frac{\bar{t}^{\prime \prime}}{\lambda}\right]$ (see Lemma 2), and (ii) we have $e_{2}^{*}\left(\underline{t}^{\prime \prime}, \bar{t}^{\prime \prime}\right) \in$ $E_{2}\left(\underline{t}^{\prime \prime}, \bar{t}^{\prime \prime}\right)$ and $e_{1}^{*}\left(\underline{t}^{\prime \prime}, \bar{t}^{\prime \prime}\right) \in E_{1}\left(\underline{t}^{\prime \prime}, \bar{t}^{\prime \prime}\right)$ (see Proposition 1$)$. The equality $e_{1}^{*}\left(\underline{t}^{\prime \prime}, \bar{t}^{\prime \prime}\right)=e_{1}^{*}\left(t_{1}^{*}(\underline{s}, \bar{s})\right)$ follows from $t_{1}^{*}(\underline{s}, \bar{s})=\left(0, \bar{t}^{\prime \prime}\right)$ for all $\bar{s} \leq s_{I}$. Hence, $t_{1}^{*}(\underline{s}, \bar{s})$ generates a lower social surplus than $t_{2}^{*}(\underline{s}, \bar{s})$; implying a lower profit for the principal because both contracts yield the agent the same equilibrium payoff of $\bar{t}^{\prime}$. To summarize:

Lemma $8 D(\underline{s}, \bar{s})<0$ if both $\bar{s} \leq s_{I I}$ and $\Delta s \leq \Delta t^{\prime \prime}$ hold.

Step 4. To characterize $D(\underline{s}, \bar{s})$ in the remaining parts of the parameter space, it will turn out to be useful to establish some properties of the partial derivative of $D(\underline{s}, \bar{s})$ with respect to $\bar{s}$. In particular, (25), (8), and Propositions 1-3 imply

$$
\frac{\partial D(\underline{s}, \bar{s})}{\partial \bar{s}}=e_{1}^{*}\left(t_{1}^{*}(\underline{s}, \bar{s})\right)-e_{2}^{*}\left(t_{2}^{*}(\underline{s}, \bar{s})\right)
$$

where obtaining (26) is straightforward because either (a) the optimal contract is derived from the respective first-order condition (in which case the Envelope Theorem applies), or (b) the optimal contract is a corner solution (and hence invariant in $s$ ). The proof of the following lemma can be found at the end of this Section.

Lemma 9 Suppose $\Delta s<\Delta s_{I I}$ holds. For any given $\underline{s}$, there exists a threshold level $\widetilde{s}(\underline{s})$ such that

$$
\frac{\partial D(\underline{s}, \bar{s})}{\partial \bar{s}}>0 \text { if } \bar{s}<\widetilde{s}(\underline{s}) \text {, and } \frac{\partial D(\underline{s}, \bar{s})}{\partial \bar{s}} \leq 0 \text { if } \bar{s} \geq \widetilde{s}(\underline{s}) .
$$

where (i) if $\underline{s} \geq s_{I I}-\Delta s_{I I}$, we have $\widetilde{s}(\underline{s})=\underline{s}+\Delta s_{I I}$, and (ii) if $\underline{s} \leq s_{I I}-\Delta s_{I I}$, we have that $\widetilde{s}(\underline{s})$ is a strictly increasing function of $\underline{s}$ that satisfies $\widetilde{s}(\underline{s}=\lambda)<\lambda+\Delta s_{I I}$ and $\widetilde{s}(\underline{s}=$ $\left.s_{I I}-\Delta s_{I I}\right)=s_{I I}$.

Now, consider the parameter region where $\bar{s} \leq s_{I I}, \Delta s \in\left(\Delta t^{\prime \prime}, \Delta s_{I I}\right)$, and $\underline{s} \in\left(s_{I I}-\right.$ $\Delta s_{I I}, s_{I I}-\Delta t^{\prime \prime}$ ) hold (for an illustration, see again Figure 12). In this case, $D(\underline{s}, \bar{s})<0$ because, for given $\underline{s}$, Lemma 7 implies $D\left(\underline{s}, s_{I I}\right)<0$, while Lemma 9 implies $\frac{\partial D(\underline{s}, \bar{s})}{\partial \bar{s}}>0$ for all $\bar{s} \leq s_{I I}$.

Lemma $10 D(\underline{s}, \bar{s})<0$ if $\bar{s} \leq s_{I I}, \Delta s \in\left(\Delta t^{\prime \prime}, \Delta s_{I I}\right)$, and $\underline{s} \in\left(s_{I I}-\Delta s_{I I}, s_{I I}-\Delta t^{\prime \prime}\right)$ hold. 
Step 5. To characterize $D(\underline{s}, \bar{s})$ in the remainder of the parameter space, suppose for a moment that $\underline{s} \leq s_{I I}-\Delta s_{I I}$ holds. In this case, (i) $D(\underline{s}, \bar{s})<0$ if $\Delta s \leq \Delta t^{\prime \prime}$ (Lemma 8), (ii) $D(\underline{s}, \bar{s})>0$ if both $\Delta s \geq \Delta s_{I I}$ and $\bar{s}<s_{I I}$ (Lemma 6), and (iii) $D(\underline{s}, \bar{s})=0$ if $\bar{s} \geq s_{I I}$ (Lemma 7). These observations in combination with Lemma 9 imply that for any $\underline{s} \leq s_{I I}-\Delta s_{I I}$ there exists a unique threshold value $B(\underline{s})$ such that (a) $D(\underline{s}, \bar{s})<0$ holds for all $\bar{s}<B(\underline{s})$, and (b) $D(\underline{s}, \bar{s}) \geq 0$ holds for all $\bar{s} \geq B(\underline{s})$. The above observations also imply that, for $\underline{s}<s_{I I}-\Delta s_{I I}$, the boundary function $B(\underline{s})$ is located in a part of the parameter space where $\Delta s \in\left(\Delta t^{\prime \prime}, \Delta s_{I I}\right)$ and $\frac{\partial D(\underline{s}, \bar{s})}{\partial \bar{s}}>0$ hold; implying that $B(\underline{s})<\widetilde{s}(\underline{s})$ for all $\underline{s}<s_{I I}-\Delta s_{I I}{ }^{29}$ With respect to its slope, note that $B(\underline{s})$ is implicitly defined by $D(\underline{s}, \bar{s})=0$. Hence, the Implicit Function Theorem implies

$$
\frac{\partial B(\underline{s})}{\partial \underline{s}}=-\frac{\frac{\partial D(\underline{s}, \bar{s})}{\partial \underline{s}}}{\frac{\partial D(\underline{s}, \bar{s})}{\partial \bar{s}}}>0 \text { for } \underline{s}<s_{I I}-\Delta s_{I I} .
$$

The inequality in (27) follows from (a) $\frac{\partial D(s, \bar{s})}{\partial \bar{s}}>0$ (see the reasoning above) and (b)

$$
\frac{\partial D(\underline{s}, \bar{s})}{\partial \underline{s}}=-\left(1-e_{2}^{*}\left(t_{2}^{*}(\underline{s}, \bar{s})\right)\right)<0
$$

where this partial derivative can be derived in a similar way as (26), and where the inequality in (28) follows from $e_{2}^{*}\left(t_{2}^{*}(\underline{s}, \bar{s})\right)<1$ for all $\Delta s<\Delta s_{I I}$ (see Proposition 2, and recall the above reasoning that, for $\underline{s}<s_{I I}-\Delta s_{I I}$, the boundary $B(\underline{s})$ is located in a part of the parameter space where $\Delta s \in\left(\Delta t^{\prime \prime}, \Delta s_{I I}\right)$ holds $)$.

Step 6. To summarize, Lemmas 6-10 and Step 5 imply that the claims in Proposition 4 hold true. In particular, the continuous boundary function $B(\underline{s})$ has the following properties: (a) if $\underline{s} \geq s_{I I}-\Delta s_{I I}$, we have $B(\underline{s})=\underline{s}+\Delta s_{I I}$, and (b) if $\underline{s}<s_{I I}-\Delta s_{I I}$, then $B(\underline{s})$ is strictly increasing in $\underline{s}$ and it is located in a part of the parameter space where $\Delta s \in\left(\Delta t^{\prime \prime}, \Delta s_{I I}\right)$ holds.

It only remains to show that contracts $t \in T_{0}$ can never be optimal. To see this, note that any contract $t \in T_{0}$ induces the agent to leave in both states (resulting in a payoff of zero for the principal). Moreover, note that the contract $\left(\bar{t}^{\prime}, \bar{t}^{\prime}\right) \in T_{2}$ would yield the principal a payoff of $\underline{s}-U_{0}$ (because $\Delta t=0$ implies $e^{*}(t)=0$ and $\bar{t}^{\prime}=U_{0}$ holds (see Proposition 1 and Definition 1 )), where $\underline{s}-U_{0}>\underline{s}-\lambda>0$ (which follows from (5) and our assumption that $\lambda<\underline{s}$ ). Hence, for all $(\underline{s}, \bar{s})$, there exists some no-separation contract (for example, the one discussed above) that yields the principal a strictly positive payoff, which implies that choosing some $t \in T_{0}$ can never be optimal from the principal's perspective.

\footnotetext{
${ }^{29}$ Note that for $\underline{s}=s_{I I}-\Delta s_{I I}$, we have $B(\underline{s})=s_{I I}$.
} 


\section{D.1 Proof of Lemma 9}

It follows from (26) that the sign of $\frac{\partial D(\underline{s}, \bar{s})}{\partial \bar{s}}$ depends on the sign of $\left[e_{1}^{*}\left(t_{1}^{*}(\underline{s}, \bar{s})\right)-e_{2}^{*}\left(t_{2}^{*}(\underline{s}, \bar{s})\right)\right]$, which we study in the following. We proceed in two steps.

Step 1. Consider the range of parameters where $\Delta s \leq \Delta s_{I}$ is satisfied, where it will turn out that $e_{2}^{*}\left(t_{2}^{*}(\underline{s}, \bar{s})\right)<e_{1}^{*}\left(t_{1}^{*}(\underline{s}, \bar{s})\right)$ holds. To see this, consider the contract $\left(\underline{t}^{\prime \prime}, \bar{t}^{\prime \prime}\right)$, where $\underline{t}^{\prime \prime} \equiv \bar{t}^{\prime \prime}-\Delta t^{\prime \prime}$ (for an illustration, see Figure 5). Under this contract, it follows from Definition 1 and Proposition 1 that the agent is indifferent between choosing any of the effort levels $e_{2}^{*}\left(\underline{t}^{\prime \prime}, \bar{t}^{\prime \prime}\right)$, $e_{1}^{*}\left(\underline{t}^{\prime \prime}, \bar{t}^{\prime \prime}\right)$, and $\min (\lambda, 1)$, where $e_{2}^{\prime \prime} \equiv e_{2}^{*}\left(\underline{t}^{\prime \prime}, \bar{t}^{\prime \prime}\right)<e_{1}^{*}\left(\underline{t}^{\prime \prime}, \bar{t}^{\prime \prime}\right) \equiv e_{1}^{\prime \prime}$ (because (i) $\bar{t}^{\prime \prime}<\lambda$ implies $E_{2}\left(\underline{t}^{\prime \prime}, \bar{t}^{\prime \prime}\right)=\left[0, \frac{\underline{t}^{\prime \prime}}{\lambda}\right]$ and $E_{1}\left(\underline{t}^{\prime \prime}, \bar{t}^{\prime \prime}\right)=\left(\frac{\underline{t}^{\prime \prime}}{\lambda}, \frac{\bar{t}^{\prime \prime}}{\lambda}\right]$ (see Lemma 2), and (ii) we have $e_{2}^{\prime \prime} \in E_{2}\left(\underline{t}^{\prime \prime}, \bar{t}^{\prime \prime}\right)$ and $e_{1}^{\prime \prime} \in E_{1}\left(\underline{t}^{\prime \prime}, \bar{t}^{\prime \prime}\right)$ (see Proposition 1). Now, note that Proposition 3 implies $e_{1}^{\prime \prime} \leq e_{1}^{*}\left(t_{1}^{*}(\underline{s}, \bar{s})\right.$ ). Moreover, from Proposition 2 it follows that for $\Delta s \leq \Delta s_{I}$ we have $e_{2}^{*}\left(t_{2}^{*}(\underline{s}, \bar{s})\right) \leq e_{2}^{\prime \prime}$, which together with the previous observations implies $e_{2}^{*}\left(t_{2}^{*}(\underline{s}, \bar{s})\right) \leq e_{2}^{\prime \prime}<e_{1}^{\prime \prime} \leq e_{1}^{*}\left(t_{1}^{*}(\underline{s}, \bar{s})\right)$, which proves the claim $e_{2}^{*}\left(t_{2}^{*}(\underline{s}, \bar{s})\right)<e_{1}^{*}\left(t_{1}^{*}(\underline{s}, \bar{s})\right)$ (implying $\frac{\partial D(s, \bar{s})}{\partial \bar{s}}>0$ in this range).

Step 2. Consider the (remaining) range of parameters where $\Delta s \in\left(\Delta s_{I}, \Delta s_{I I}\right)$ holds. First, suppose that, in addition, $\bar{s} \geq s_{I I}$ holds. In this case, we have $e_{2}^{*}\left(t_{2}^{*}(\underline{s}, \bar{s})\right)<e_{1}^{*}\left(t_{1}^{*}(\underline{s}, \bar{s})\right)=1$ (see Propositions 2 and 3), which implies $\frac{\partial D(s, \bar{s})}{\partial \bar{s}}>0$ in this range of parameters. Second, note that, in the region of the $(\underline{s}, \bar{s})$-space where both $\Delta s \in\left(\Delta s_{I}, \Delta s_{I I}\right)$ and $\bar{s} \in\left[s_{I}, s_{I I}\right)$ hold, $e_{1}^{*}\left(t_{1}^{*}(\underline{s}, \bar{s})\right)=e_{2}^{*}\left(t_{2}^{*}(\underline{s}, \bar{s})\right)$ is equivalent to

$$
\frac{1}{2}\left(\frac{\bar{s}+\lambda}{1+2 \lambda}\right)=\frac{1}{2}(\Delta s-\sqrt{1+2 \lambda}+1) \Leftrightarrow \bar{s}=\underbrace{\left(\frac{1+2 \lambda}{2 \lambda}\right)}_{>1} \cdot \underline{s}+\underbrace{\left(\frac{\lambda+(1+2 \lambda)(\sqrt{1+2 \lambda}-1)}{2 \lambda}\right)}_{>0},
$$

which follows from Propositions 1, 2, and 3 .

To summarize what we have learned from Steps 1 and 2 sofar, in the parameter region where both $\Delta s<\Delta s_{I I}$ and $\bar{s} \geq s_{I}$ hold, the boundary function $\widetilde{s}(\underline{s})$ is defined (a) by $\widetilde{s}(\underline{s})=\underline{s}+\Delta s_{I I}$ if $\underline{s} \geq s_{I I}-\Delta s_{I I}$, and (b) by (29) (and located as depicted in Figure 12) if $\underline{s} \leq s_{I I}-\Delta s_{I I}$.

Finally, consider the region of the $(\underline{s}, \bar{s})$-space, where both $\Delta s \in\left(\Delta s_{I}, \Delta s_{I I}\right)$ and $\bar{s}<s_{I}$ hold (where such $(\underline{s}, \bar{s})$ may or may not exist). In this region of the parameter space, $e_{1}^{*}\left(t_{1}^{*}(\underline{s}, \bar{s})\right)=$ $e_{2}^{*}\left(t_{2}^{*}(\underline{s}, \bar{s})\right)$ is equivalent to (see Propositions 1,2 , and 3 ):

$$
\frac{1}{2}\left(\frac{\left(2 \bar{t}^{\prime \prime}+\lambda\right)+\lambda}{1+2 \lambda}\right)=\frac{1}{2}(\Delta s-\sqrt{1+2 \lambda}+1)
$$


and hence, in this parameter range, the boundary function $\widetilde{s}(\underline{s})$ is a line parallel to the main diagonal, where $e_{1}^{*}\left(t_{1}^{*}(\underline{s}, \bar{s})\right)>(<) e_{2}^{*}\left(t_{2}^{*}(\underline{s}, \bar{s})\right)$ to the left (right) of this line. Moreover, independent of the existence of pairs $(\underline{s}, \bar{s})$ that satisfy both $\Delta s \in\left(\Delta s_{I}, \Delta s_{I I}\right)$ and $\bar{s}<s_{I}$, the above discussion implies that, for $\underline{s} \leq s_{I I}-\Delta s_{I I}$, the boundary function $\widetilde{s}(\underline{s})$ is (piece-wise) linear and increasing in $\underline{s}$, and it satisfies $\widetilde{s}(\underline{s}=\lambda)<\lambda+\Delta s_{I I}$ and $\widetilde{s}\left(\underline{s}=s_{I I}-\Delta s_{I I}\right)=s_{I I}$, which concludes the proof.

\section{E Proof of Proposition 6}

In the proof of Proposition 6, we maintain the following two assumptions. First, if, at date 4, the agent is indifferent between leaving and staying, he stays (see footnote 10). Second, consider a given pair of contracts and suppose that both generate the same effort incentives (i.e., given truth-telling and taking into account optimal ex-post participation decisions, the agent's payoff as a function of his effort choice $e$ is the same under both contracts). Then, the agent chooses the same effort level under both contracts (i.e., potentially arising indifferences are resolved in the same way).

For a given contract $\left(\tau(\widehat{e}, \widehat{s} ; s), \tau_{0}(\widehat{e}, \widehat{s})\right)$ in $T$ define sets of effort levels $E^{S}(s) \equiv\{e /$ given $e$ and $s$, in equilibrium the agent stays $\}$ and $E^{L}(s) \equiv\{e /$ given $e$ and $s$, in equilibrium the agent leaves\}. We first establish the following result, which proves the first part of Proposition 6.

Lemma 11 (Restriction to $\widetilde{T}$ is without loss of generality) For any $\operatorname{contract}(\tau(\widehat{e}, \widehat{s} ; s)$, $\left.\tau_{0}(\widehat{e}, \widehat{s})\right)$ in $T$, there exist threshold values $\widetilde{e}(\underline{s}), \widetilde{e}(\bar{s}) \in \Re$ and payments $\widetilde{\tau}(\underline{s}), \widetilde{\tau}(\bar{s}), \widetilde{\tau}_{0} \geq 0$ satisfying $\widetilde{\tau}(s)=\lambda \widetilde{e}(s)+\widetilde{\tau}_{0}$ for all $s \in\{\underline{s}, \bar{s}\}$ (where $\widetilde{e}()$ and $\widetilde{\tau}()$ are functions of the verifiable gross profit only) such that

(i) for s given, (a) if $e \in E^{S}(s)$, then $e \leq \widetilde{e}(s)$ and the agent receives the payment $\widetilde{\tau}(s)$, and (b) if $e \in E^{L}(s)$, then $e \geq \widetilde{e}(s)$ and the agent receives the payment $\widetilde{\tau}_{0}$, and

(ii) the message-independent contract $\left(\widetilde{\tau}(\underline{s}), \widetilde{\tau}(\bar{s}), \widetilde{\tau}_{0}\right)$ leads to the same equilibrium outcome.

The proof of Lemma 11 proceeds in five steps. In Steps 1-3 we establish some preliminary results, which are used in Steps 4-5 to establish Lemma 11. 
Step 1. Take $s$ as given. We first show that for all $e \in E^{S}(s)$ the equilibrium payment $\tau(e, s ; s)$ cannot vary in $e$. This is trivially satisfied if $E^{S}(s)$ is a singleton, and hence suppose that this is not the case. For any pair $e^{\prime}, e^{\prime \prime} \in E^{S}(s),(13)$ implies that both

$$
\begin{aligned}
\max \left\{\tau\left(e^{\prime}, s, s\right), \lambda e^{\prime}+\tau_{0}\left(e^{\prime}, s\right)\right\} & \geq \tau\left(e^{\prime \prime}, s, s\right) \Leftrightarrow \tau\left(e^{\prime}, s, s\right) \geq \tau\left(e^{\prime \prime}, s, s\right) \text { and } \\
\max \left\{\tau\left(e^{\prime \prime}, s, s\right), \lambda e^{\prime \prime}+\tau_{0}\left(e^{\prime \prime}, s\right)\right\} & \geq \tau\left(e^{\prime}, s, s\right) \Leftrightarrow \tau\left(e^{\prime \prime}, s, s\right) \geq \tau\left(e^{\prime}, s, s\right)
\end{aligned}
$$

have to hold, which can only be satisfied if $\tau\left(e^{\prime \prime}, s, s\right)=\tau\left(e^{\prime}, s, s\right)$. To summarize, $\tau(e, s ; s) \equiv$ $\widetilde{\tau}(s)$ for all $e \in E^{S}(s)$, where $\widetilde{\tau}(s)$ may vary in the realized, verifiable gross profit $s \in\{\underline{s}, \bar{s}\}$.

Step 2. Take $s$ as given. An analogous argument as in Step 1 implies that for all $e \in E^{L}(s)$ the equilibrium payment $\tau_{0}(e, s)$ cannot vary in $e$, i.e., $\tau_{0}(e, s) \equiv x_{0}(s)$ for all $e \in E^{L}(s)$, where (so far) we do not ruled out that the equilibrium payment $x_{0}(s)$ may depend on the agent's report about the realized state.

Step 3. For $s$ given, suppose $E^{S}(s), E^{L}(s) \neq \varnothing$. For this case, we show that truth-telling implies that there exists a threshold value $\varepsilon(s)$ such that (i) $e \in E^{S}(s)$ implies $e \leq \varepsilon(s)$, and (ii) $e \in E^{L}(s)$ implies $e \geq \varepsilon(s)$, where $\varepsilon(s)$ is implicitly defined by

$$
\widetilde{\tau}(s)=\lambda \cdot \varepsilon(s)+x_{0}(s)
$$

First, consider $E^{L}(s)$. If $\varepsilon(s) \leq 0$ would hold, then the claim with respect to $E^{L}(s)$ would be trivially satisfied. Hence, suppose $\varepsilon(s)>0$, and, contrary to the above claim, assume that there exist $e^{\prime} \in E^{L}(s)$ such that $e^{\prime}<\varepsilon(s)$; implying $\max \left\{\tau\left(e^{\prime}, s, s\right), \lambda e^{\prime}+\tau_{0}\left(e^{\prime}, s\right)\right\}=\lambda e^{\prime}+x_{0}(s)<$ $\widetilde{\tau}(s)$, where the equality follows from $e^{\prime} \in E^{L}(s)$ and where the inequality follows from $e^{\prime}<\varepsilon(s)$ (which is equivalent to $\widetilde{\tau}(s)>\lambda e^{\prime}+x_{0}(s)$ ). Hence, if $e^{\prime}<\varepsilon(s)$ would hold, the agent's incentive compatibility condition (13) would be violated, because the agent could obtain the larger payoff of $\widetilde{\tau}(s)$ by, instead of truthfully reporting $e^{\prime}$, switch to some false report $e^{\prime \prime} \in E^{S}(s)$. Hence, $e \geq \varepsilon(s)$ for all $e \in E^{L}(s)$. Second, consider $E^{S}(s)$. A completely analogous argument (which we, therefore, omit) implies the claim with respect to $E^{S}(s)$.

Step 4. Building on Steps 1-3, we now prove Lemma 11 for the case that, for all $s$, it holds that $E^{S}(s), E^{L}(s) \neq \varnothing$. For this case, we first establish that $x_{0}(s) \equiv \widetilde{\tau}_{0}$ has to hold for all $s \in\{\underline{s}, \bar{s}\}$. To see this, note that in the present case $\left\{E^{L}(\underline{s}) \cap E^{L}(\bar{s})\right\} \neq \varnothing$ (see the discussion of the threshold values $\varepsilon(s)$ in Step 3), and (13) in combination with Step 2 implies that for all $e \in\left\{E^{L}(\underline{s}) \cap E^{L}(\bar{s})\right\}$ both $\lambda e+x_{0}(\underline{s}) \geq \lambda e+x_{0}(\bar{s})$ and $\lambda e+x_{0}(\bar{s}) \geq \lambda e+x_{0}(\underline{s})$ have to 
hold. This can only be satisfied if $x_{0}(s) \equiv \widetilde{\tau}_{0}$ for all $s \in\{\underline{s}, \bar{s}\}$. This observation implies that, in the present case, we indeed have $\varepsilon(s)=\widetilde{e}(s) \forall s$ (where $\widetilde{e}(s)$ is defined in Lemma 11). For the present case, these observations prove part (i) of Lemma 11. Moreover, these observations imply that both the contract $\left(\tau(\widehat{e}, \widehat{s} ; s), \tau_{0}(\widehat{e}, \widehat{s})\right)$ and the contract $\left.\left(\widetilde{\tau}(\underline{s}), \widetilde{\tau}(\bar{s}), \widetilde{\tau}_{0}\right)\right)$ imply the same sets $E^{S}(s)$ and $E^{L}(s)$ for all $s \in\{\underline{s}, \bar{s}\}$. Hence, for all $e$ and all $s$, both contracts lead to the same ex-post participation decisions and payments to the agent; implying that the agent's effort incentives under both contracts are identical. As a result, both contracts lead to the same equilibrium outcome, which, for the present case, proves part (ii) of Lemma 11.

Step 5. Building on Steps 1-3, we now prove Lemma 11 for the remaining cases (where $E^{S}(s), E^{L}(s) \neq \varnothing$ does not hold for all $\left.s\right)$. For each of the remaining cases, we derive some observations with respect to the threshold values $\widetilde{e}(\underline{s})$ and $\widetilde{e}(\bar{s})$ and the payments $\widetilde{\tau}(\underline{s}), \widetilde{\tau}(\bar{s})$, and $\widetilde{\tau}_{0}$. These observations in combination with a similar reasoning as in Step 4 will imply the Proposition for the respective case, where, due to its similarity to Step 4, this latter step is, however, omitted from the proof. First, suppose $E^{L}(\underline{s})=E^{L}(\bar{s})=[0,1]$, where a similar reasoning as in Step 4 implies that $x_{0}(\underline{s})=x_{0}(\bar{s}) \equiv \widetilde{\tau}_{0}>0$ has to hold. Additionally, define $\widetilde{\tau}(s) \equiv 0$ for all $s$, and implicitly define $\widetilde{e}(s)$ by $0=\lambda \widetilde{e}(s)+\widetilde{\tau}_{0}$ for all $s$, where, due to $\widetilde{\tau}_{0}>0$, we have $\widetilde{e}(s)<0$. Second, suppose $E^{S}(\underline{s})=E^{S}(\bar{s})=[0,1]$; implying that, depending on the realized state, the agent receives a payment $\widetilde{\tau}(\underline{s})$ or $\widetilde{\tau}(\bar{s})$. Additionally, define $\widetilde{\tau}_{0} \equiv 0$, in which case $\widetilde{e}(s)$ is implicitly defined by $\widetilde{\tau}(s)=\lambda \widetilde{e}(s)$ for all $s$, where due to $E^{S}(\underline{s})=E^{S}(\bar{s})=[0,1]$ we have $\widetilde{e}(s) \geq 1$. Third, suppose $E^{L}(\underline{s})=E^{S}(\bar{s})=[0,1]$; implying that in state $\bar{s}$ the agent receives a payment $\widetilde{\tau}(\bar{s})$, while in state $\underline{s}$ he receives $x_{0}(\underline{s})$, where $x_{0}(\underline{s})>0$. In this case, $(13)$ in combination with Steps 1 and 2 implies that, given the contract $\left(\tau(\widehat{e}, \widehat{s} ; s), \tau_{0}(\widehat{e}, \widehat{s})\right)$, it must be the case that $\widetilde{\tau}(\bar{s}) \geq \lambda e+x_{0}(\underline{s})$ for all $e$. Hence, define $\widetilde{\tau}(\underline{s}) \equiv 0$ and $\widetilde{\tau}_{0} \equiv x_{0}(\underline{s})$; implying $\widetilde{e}(\underline{s})<$ 0 and $\widetilde{e}(\bar{s}) \geq 1$. The proof for the case $E^{L}(\bar{s})=E^{S}(\underline{s})=[0,1]$ is completely analogous and therefore omitted. Fourth, suppose $E^{L}(\underline{s}) \subset[0,1]=E^{S}(\bar{s})$. In this case, (13) in combination with Steps 1 and 2 again implies that, under the contract $\left(\tau(\widehat{e}, \widehat{s} ; s), \tau_{0}(\widehat{e}, \widehat{s})\right)$, it must be the case that $\widetilde{\tau}(\bar{s}) \geq \lambda e+x_{0}(\underline{s})$ holds for all $e$. Now, define $\widetilde{\tau}_{0} \equiv x_{0}(\underline{s})$, which in combination with $\widetilde{\tau}(\bar{s}) \geq \lambda e+\widetilde{\tau}_{0} \forall e$ implies $\widetilde{e}(\bar{s}) \geq 1$. Also, as in the present case, in state $\underline{s}$ the agent leaves for some $e$ but stays for others, Steps 3 and 4 imply that $\widetilde{e}(\underline{s})=\varepsilon(\underline{s}) \in[0,1)$. The proof for the case $E^{L}(\bar{s}) \subset[0,1]=E^{S}(\underline{s})$ is completely analogous and therefore omitted. Finally, suppose $E^{S}(\bar{s}) \subset[0,1]=E^{L}(\underline{s})$. In this case, it follows from Step 4 that $x_{0}(\bar{s})=x_{0}(\underline{s}) \equiv \widetilde{\tau}_{0}>0$. Define 
$\widetilde{\tau}(\underline{s}) \equiv 0$, and hence $\lambda \widetilde{e}(\underline{s})+\widetilde{\tau}_{0}=0$ implies $\widetilde{e}(\underline{s})=\varepsilon(\underline{s})<0$. Also, it follows from Steps 3 and 4 that $E^{S}(\bar{s}) \subset[0,1]$ implies $\widetilde{e}(\bar{s})=\varepsilon(\bar{s}) \in[0,1)$. The proof for the case $E^{S}(\underline{s}) \subset[0,1]=E^{L}(\bar{s})$ is completely analogous and therefore omitted. This concludes the proof of Lemma 11.

We now turn to the proof of the second part of Proposition 6, where the proof consists of two parts (Lemmas 12 and 13).

Lemma 12 (No strictly positive payment when the gross profit is zero) For any contract $\left(\widetilde{\tau}(\underline{s}), \widetilde{\tau}(\bar{s}), \widetilde{\tau}_{0}\right)$ in $\widetilde{T}$ satisfying $\widetilde{\tau}_{0}>0$ there exists an alternative contract in $\widetilde{T}$ that specifies a payment of zero when the gross profit is zero and that yields the principal a strictly higher payoff.

The proof of Lemma 12 proceeds in two steps. In Step 1, we show that, if $\widetilde{\tau}_{0}>0$ holds, without loss of generality, one can restrict attention to contracts satisfying $\widetilde{\tau}(s) \geq \widetilde{\tau}_{0}$ for all $s$. Building on this result, in Step 2 we show that any such contract is dominated by some contract specifying $\widetilde{\tau}_{0}=0$.

Step 1. Take a contract $\left(\widetilde{\tau}(\underline{s}), \widetilde{\tau}(\bar{s}), \widetilde{\tau}_{0}\right)$ with $\widetilde{\tau}_{0}>0$ as given and suppose that there exists some $s$ such that $\widetilde{\tau}(s)<\widetilde{\tau}_{0}$. Without loss of generality, suppose $\widetilde{\tau}(\underline{s})<\widetilde{\tau}_{0}$, and consider the alternative contract $\left(\widetilde{\tau}_{0}, \widetilde{\tau}(\bar{s}), \widetilde{\tau}_{0}\right)$. In state $\bar{s}$, under both the original and the alternative contract the agent's ex-post participation behavior and the payoffs he obtains are exactly the same (because the specified payments are exactly the same). In state $\underline{s}$, under the original contract, for all $e$, the agent leaves (because $\widetilde{\tau}(\underline{s})<\lambda e+\widetilde{\tau}_{0} \forall e$ ), and he receives a payment of $\widetilde{\tau}_{0}$. Under the alternative contract, we have $\widetilde{\tau}_{0}<(=) \lambda e+\widetilde{\tau}_{0}$ for $e>0(e=0)$, and hence (as under the original contract) the agent leaves and receives $\widetilde{\tau}_{0}$ for all $e>0$. For $e=0$, under the alternative contract the agent would stay (because he is indifferent), but again receive $\widetilde{\tau}_{0}$. Consequently, from the principal's perspective, both contracts generate the same effort incentives and payments to the agent. Moreover, if $e=0$ would indeed be chosen in equilibrium, the principal would be better off under the alternative contract because it induces participation. Consequently, without loss of generality one can restrict attention to contracts $\left(\widetilde{\tau}(\underline{s}), \widetilde{\tau}(\bar{s}), \widetilde{\tau}_{0}\right)$ satisfying $\widetilde{\tau}(s)-\widetilde{\tau}_{0} \geq 0$ for all $s$.

Step 2. Take a contract $\left(\widetilde{\tau}(\underline{s}), \widetilde{\tau}(\bar{s}), \widetilde{\tau}_{0}\right)$ with $\widetilde{\tau}_{0}>0$ as given and suppose $\widetilde{\tau}(s)-\widetilde{\tau}_{0} \geq 0$ for all $s$. First, consider the agent's ex-post participation decision. For given $e$, the agent stays with the principal if $\widetilde{\tau}(s) \geq \lambda e+\widetilde{\tau}_{0} \Leftrightarrow \widetilde{\tau}(s)-\widetilde{\tau}_{0} \geq \lambda e$ holds. Now, define an alternative 
contract $(\underline{t}, \bar{t}, 0)$, where $\underline{t} \equiv \widetilde{\tau}(\underline{s})-\widetilde{\tau}_{0} \geq 0$ and $\bar{t} \equiv \widetilde{\tau}(\bar{s})-\widetilde{\tau}_{0} \geq 0$. This alternative contract yields the same ex-post participation behavior as the original contract $\left(\widetilde{\tau}(\underline{s}), \widetilde{\tau}(\bar{s}), \widetilde{\tau}_{0}\right)$. Second, consider the agent's effort incentives. When choosing his effort level, under the original contract $\left(\widetilde{\tau}(\underline{s}), \widetilde{\tau}(\bar{s}), \widetilde{\tau}_{0}\right)$, the agent's objective function is given by

$$
e \cdot \max \left(\widetilde{\tau}(\bar{s}), \lambda e+\widetilde{\tau}_{0}\right)+(1-e) \cdot \max \left(\widetilde{\tau}(\underline{s}), \lambda e+\widetilde{\tau}_{0}\right)-c(e)
$$

Under the alternative contract $(\underline{t}, \bar{t}, 0)$, in each contingency the payment to the agent is reduced by $\widetilde{\tau}_{0}$, and hence the agent's objective function can be written as

$$
\left[e \cdot \max \left(\widetilde{\tau}(\bar{s}), \lambda e+\widetilde{\tau}_{0}\right)+(1-e) \cdot \max \left(\widetilde{\tau}(\underline{s}), \lambda e+\widetilde{\tau}_{0}\right)-c(e)\right]-\widetilde{\tau}_{0}
$$

Hence, under both contracts the agent faces identical effort incentives and chooses the same equilibrium effort level. Third, as under both contracts choosing $e=0$ would be feasible (which in either case would yield the agent a non-negative payoff), under both contracts the agent's ex-ante participation constraint is satisfied. Finally, as under the alternative contract the expected payment to the agent is reduced by $\widetilde{\tau}_{0}>0$, the principal is strictly better off under the alternative contract. This concludes the proof of Lemma 12.

Lemma 12 shows that, without loss of generality, one can restrict attention to contracts in $\widetilde{T}$ that satisfy $\widetilde{\tau}_{0}=0$. Now, consider contracts $\left(\widetilde{\tau}(\underline{s}), \widetilde{\tau}(\bar{s}), \widetilde{\tau}_{0}\right)$ in $\widetilde{T}$ that satisfy $\widetilde{\tau}(\underline{s})>\widetilde{\tau}(\bar{s}) \geq$ $\widetilde{\tau}_{0}=0$, and denote this subset of contracts by $T^{-}$.

Lemma 13 (No reward for low gross profit) For any contract in $T^{-}$, there exists an alternative contract $\left(\widetilde{\tau}(\underline{s}), \widetilde{\tau}(\bar{s}), \widetilde{\tau}_{0}\right)$ in $\widetilde{T}$ that satisfies $\widetilde{\tau}(\bar{s}) \geq \widetilde{\tau}(\underline{s}) \geq \widetilde{\tau}_{0}=0$ and that yields the principal a strictly higher payoff.

In order to prove Lemma 13, we first characterize optimal agent behavior across $T^{-}$, then derive optimal contracts in $T^{-}$, and subsequently we show that contracts in $T^{-}$are strictly dominated. For ease of notation (and consistent with the main body of the paper), define $\underline{t} \equiv \widetilde{\tau}(\underline{s}), \bar{t} \equiv \widetilde{\tau}(\bar{s})$, and $t \equiv(\underline{t}, \bar{t})$. Applying an analogous reasoning as that above Lemma 2 (see Section 3), it is straightforward to show that, for a given contract $t \in T^{-}$and optimal ex-post participation decisions, the agent's expected payoff is given by

$$
U(e, t)= \begin{cases}U_{2}^{-}(e, t) \equiv e \cdot \bar{t}+(1-e) \cdot \underline{t}-\frac{1}{2} e^{2} & \text { if } e \in E_{2}^{-}(t) \equiv\left\{e \mid e \leq \frac{\bar{t}}{\lambda}\right\} \\ U_{1}^{-}(e, t) \equiv e \cdot \lambda e+(1-e) \cdot \underline{t}-\frac{1}{2} e^{2} & \text { if } e \in E_{1}^{-}(t) \equiv\left\{e \mid \frac{\bar{t}}{\lambda}<e \leq \frac{t}{\lambda}\right\} \\ U_{0}^{-}(e, t) \equiv \lambda e-\frac{1}{2} e^{2} & \text { if } e \in E_{0}^{-}(t) \equiv\left\{e \mid e>\frac{t}{\lambda}\right\},\end{cases}
$$


where (a) $E_{2}^{-}(t), E_{1}^{-}(t), E_{0}^{-}(t) \neq \varnothing$ for $\underline{t}<\lambda$, (b) $E_{2}^{-}(t), E_{1}^{-}(t) \neq \varnothing$ and $E_{0}^{-}(t)=\varnothing$ for $\bar{t}<\lambda \leq \underline{t}$, and (c) $E_{2}^{-}(t) \neq \varnothing$ and $E_{1}^{-}(t)=E_{0}^{-}(t)=\varnothing$ for $\bar{t} \geq \lambda$. Moreover, note that, for $t \in T^{-}, U(e, t)$ is continuous in $e, U_{2}^{-}(e, t)$ and $U_{0}^{-}(e, t)$ are strictly concave in $e$, and $U_{1}^{-}(e, t)$ is strictly concave in $e$ if and only if $\lambda<\frac{1}{2}$. Hence, for any $t \in T^{-}$the agent either stays in both states, leaves in both states, or stays in the bad state $\underline{s}$ only.

We now turn to the agent's optimal choice of effort level given a contract $t \in T^{-}$. Note that $U_{2}^{-}(e, t)$ is strictly decreasing in $e$, and hence in the set $E_{2}^{-}(t)$ the optimal effort level is given by $e=0$; implying that the agent's maximum payoff in $E_{2}^{-}(t)$ is given by $U_{2}^{-}(0, t)=\underline{t}$.

Next, we show that $E_{1}^{-}(t) \neq \varnothing$ implies $e^{*}(t) \notin E_{1}^{-}(t)$. To prove this, we show that $U_{1}^{-}(e, t)<\underline{t}$ for all $e \in E_{1}^{-}(t)$ (where, recall, $\underline{t}$ is the payoff the agent could obtain in $E_{2}^{-}(t)$ ). On the one hand, note that $U_{1}^{-}(e, t)=\left(\lambda-\frac{1}{2}\right) e^{2}-e \underline{t}+\underline{t}<\underline{t}$ if $\lambda-\frac{1}{2}<0$ (because $e>0$ for all $e \in E_{1}^{-}(t)$, which follows from (36)). On the other hand, if $\lambda-\frac{1}{2} \geq 0$ (i.e., when $U_{1}^{-}(e, t)$ is weakly convex in $e$ ), then the continuity of $U(e, t)$ implies that, for the claim to hold true, it suffices to check whether the claim holds at the upper bound of $E_{1}^{-}(t)$. This is indeed the case because $U_{1}^{-}\left(\frac{t}{\lambda}, t\right)=\underline{t}-\frac{1}{2}\left(\frac{t}{\lambda}\right)^{2}<\underline{t}$, which follows from $\underline{t}>\bar{t} \geq 0$ (see the definition of $T^{-}$).

The fact that $e^{*}(t) \notin E_{1}^{-}(t)$ if $E_{1}^{-}(t) \neq \varnothing$ in combination with the above stated properties of $U(e, t)$ (see (36)) implies that $e^{*}(t)=0 \in E_{2}^{-}(t)$ for all $t \in T^{-}$that satisfy $\underline{t} \geq \lambda$. Moreover, for the case $\underline{t}<\lambda$ it is straightforward to show that the optimal effort level in $E_{2}^{-}(t)$ yields the agent a higher (lower) payoff than the optimal effort level in $E_{0}^{-}(t)$ if $\underline{t}>(<) U_{0}$ holds. Note that $U_{0}<\lambda$. These observations imply that, for $t \in T^{-}$, we have $e^{*}(t)=0 \in E_{2}^{-}(t)$ if $\underline{t} \geq U_{0}$, and $e^{*}(t)=\min (\lambda, 1) \in E_{0}^{-}(t)$ if $\underline{t}<U_{0}$. In the latter case, the principal's payoff is equal to zero because the agent leaves in both states of the world (see (8)). Moreover, in the former case (i.e., for contracts $t \in T^{-}$satisfying $\underline{t} \geq U_{0}$ ), the above observations imply that the principal's payoff (see again (8)) is given by $\underline{s}-\underline{t}$. Hence, in this set of contracts, the principal optimally sets $\underline{t}=U_{0}$ and obtains a payoff of $\underline{s}-U_{0}$, where we have $\underline{s}-U_{0}>\underline{s}-\lambda>0$ (implying that any contract $t \in T^{-}$that induces the agent to leave in both states (resulting in a payoff of zero for the principal) can never be optimal).

Hence, to prove that contracts in $T^{-}$cannot be optimal, it remains to show that, for any $(\underline{s}, \bar{s})$, the contract that is globally optimal in the set $T_{2} \cup T_{1}$ yields the agent a payoff that is strictly larger than $\underline{s}-U_{0}$. To see why this is true note that the contract $\left(\bar{t}^{\prime}, \bar{t}^{\prime}\right) \in T_{2}$ would also yield the principal a payoff of $\underline{s}-U_{0}$ (because $\Delta t=0$ implies $e^{*}(t)=0$ and $\bar{t}^{\prime}=U_{0}$ holds (see 
Proposition 1 and Definition 1)). However, as is apparent from Proposition 2 there does not exist $(\underline{s}, \bar{s})$ such that $\left(\bar{t}^{\prime}, \bar{t}^{\prime}\right)$ is an optimal no-separation contract, which necessarily implies that, for all $(\underline{s}, \bar{s})$, the optimal no-separation contract yields the principal a strictly higher payoff. Hence, contracts in $T^{-}$can never be optimal. This concludes the proof of Lemma 13 and the proof of the second part of Proposition 6.

\section{References}

Anton, J., And D. YaO (1995): "Start-Ups, Spin-offs, and Internal Projects," Journal of Law, Economics, and Organization, 11(2), 362-378.

Dewatripont, M., I. Jewitt, and J. Tirole (1999): "The Economics of Career Concerns, Part I: Comparing Information Structures," Review of Economic Studies, 66(1), 183-198.

Drucker, P. (1959): Landmarks of Tomorrow: A Report on the New 'Post-Modern' World. Harper Colophon Books, New York.

Fudenberg, D., And J. Tirole (1990): "Moral Hazard and Renegotiation in Agency Contracts," Econometrica, 58(6), 1279-1319.

Gibbons, R., and K. Murphy (1992): "Optimal Incentive Contracts in the Presence of Career Concerns: Theory and Evidence," Journal of Political Economy, 100(3), 468-505.

Gibbons, R., and M. Waldmann (1999): "Careers in Organizations: Theory and Evidence," in Handbook of Labor Economics, ed. by O. Ashenfelter, and D. Card, vol. 3b, pp. 2373-2437. Elsevier, Amsterdam.

GiLSON, R. (1999): "The Legal Infrastructure of High Technology Industrial Districts: Silicon Valley, Route 138, and Covenants Not To Compete," New York University Law Review, $74(3), 575-629$.

Gompers, P., And J. Lerner (2000): "The Determinants of Corporate Venture Capital Success: Organizational Structure, Incentives and Complementarities," in Concentrated Corporate Ownership, ed. by R. Morck, pp. 17-50. University of Chicago Press.

Harris, M., And B. Holmstrom (1982): "A Theory of Wage Dynamics," Review of Economic Studies, 49(3), 315-333. 
Holmstrom, B. (1983): "Equilibrium Long-Term Labor Contracts," Quarterly Journal of Economics, 98, 23-54.

(1999): "Managerial Incentive Problems: A Dynamic Perspective," Review of Economic Studies, 66(1), 169-182.

Hvide, H., And E. KRistiansen (2007): "Management of Knowledge Workers," CEPR Discussion Paper No. 6039.

KrÄKel, M., And D. Sliwka (2009): "Should You Allow Your Employee To Become Your Competitor? On Noncompete Agreements In Employment Contracts," International Economic Review, 50(1), 117-141.

Krueger, D., and H. Uhlig (2006): "Competitive Risk Sharing Contracts with One-Sided Commitment," Journal of Monetary Economics, 53(7), 1661-1691.

Laffont, J.-J., And D. Martimort (2002): The Theory of Incentives: The Principal-Agent Problem. Princeton University Press, Princeton.

Lerner, J., And U. Malmendier (2010): “Contractibility and the Design of Research Agreements," American Economic Review, 100(1), 214-246.

MA, C. (1991): "Adverse Selection in Dynamic Moral Hazard," Quarterly Journal of Economics, 106(1), 255-275.

Malcomson, J. (1999): "Individual Employment Contracts," in Handbook of Labor Economics, ed. by O. Ashenfelter, and D. Card, vol. 3B, pp. 2291-2372. Elsevier, Amsterdam.

Meyer, M. (1987): "Labor Contracts under Asymmetric Information When Workers are Free to Quit," Quarterly Journal of Economics, 102(3), 527-551.

Netzer, N., And F. Scheuer (2008): "Competitive Markets without Commitment," University of Zurich, Working Paper No. 0814.

O'Flaherty, B., and A. Siow (1995): "Up-Or-Out Rules in the Market for Lawyers," Journal of Labor Economics, 13(4), 709-735. 
Pakes, A., and S. Nitzan (1983): "Optimum Contracts for Research Personnel, Research Employment, and the Establishment of "Rival" Enterprises," Journal of Labor Economics, $1(4), 345$.

Phelan, C. (1995): "Repeated Moral Hazard and One-Sided Commitment," Journal of Economic Theory, 66(2), 488-506.

Posner, E. A., G. G. Triantis, and A. J. Triantis (2004): "Investing in Human Capital: The Efficiency of Covenants Not to Compete," University of Chicago Law School, Olin Working Paper No. $13 \%$.

Rasul, I., And S. Sonderegger (2010): "The Role of the Agent's Outside Options in Principal-Agent Relationships," Games and Economic Behavior, 68, 781-788.

Sappington, D. (1983): "Limited Liability Contracts Between Principal and Agent," Journal of Economic Theory, 29(1), 1-21.

Tirole, J. (1999): "Incomplete Contracts: Where Do We Stand?," Econometrica, 67(4), $741-781$.

Walker, R. (2005): "Empirical Analysis of Up-Or-Out Rules for Promotion Policies," Journal of Economics and Finance, 29(2), 172-186.

Wickelgren, A. (2009): "The Perverse Effects of Outside Options on Strategic Delay in Bargaining," Journal of Institutional and Theoretical Economics, 165(2), 210-229. 